Meta

Journal des traducteurs

Translators' Journal

\title{
Les noms de pays, de peuples et de lieux dans le langage imagé
}

\section{Henri Van Hoof}

Volume 44, numéro 2, juin 1999

URI : https://id.erudit.org/iderudit/004546ar

DOI : https://doi.org/10.7202/004546ar

Aller au sommaire du numéro

Éditeur(s)

Les Presses de l'Université de Montréal

ISSN

0026-0452 (imprimé)

1492-1421 (numérique)

Découvrir la revue

Citer cet article

Van Hoof, H. (1999). Les noms de pays, de peuples et de lieux dans le langage imagé. Meta, 44(2), 312-370. https://doi.org/10.7202/004546ar
Résumé de l'article

Cet article traite de l'utilisation subjective/figurée des toponymes et anthroponymes dans les langues française, anglaise et allemande. L'auteur fait un tour complet du phénomène et présente ensuite trois lexiques avec explications et équivalents. d'utilisation que vous pouvez consulter en ligne.

https://apropos.erudit.org/fr/usagers/politique-dutilisation/ 


\title{
ÉTUDES TERMINOLOGIQUES ET LINGUISTIQUES
}

\section{Les noms de pays, de peuples et de lieux dans le langage imagé}

\author{
henri van hoof \\ Centre de Terminologie de Bruxelles, \\ Bruxelles, Belgique
}

\begin{abstract}
RÉSUMÉ
Cet article traite de l'utilisation subjective/figurée des toponymes et anthroponymes dans les langues française, anglaise et allemande. L'auteur fait un tour complet du phénomène et présente ensuite trois lexiques avec explications et équivalents.
\end{abstract}

\begin{abstract}
This article deals with the subjective/figurative use of toponyms and anthroponyms in English, French and German. The author describes the phenomenon and provides trilingual lexicons with explanations and equivalents.
\end{abstract}

L'emploi de noms de pays et de nationalités, de régions et de localités remplit dans la langue une double fonction, l'une objective, l'autre subjective. Dans la première, ils ne servent qu'à indiquer une provenance, une origine: dentelle de Bruxelles, bleu de Berlin, chat persan, berger allemand, encre de Chine, arbre de Judée, cochon d'Inde, havane, bourgogne, etc. Nous négligerons ici cette fonction objective pour nous intéresser exclusivement à leur utilisation subjective - c'est-à-dire figurée - et à la manière dont elle se reflète dans les langues anglaise et allemande.

\section{RÉMINISCENCES BIBLIQUES, HISTORIQUES OU LITTÉRAIRES}

Les termes et expressions inspirés de la Bible, de l'histoire ou de la littérature existent le plus souvent sous une forme identique ou très proche dans les trois langues: regretter les oignons d'Égypte, to sigh for the fleshpots of Egypt, sich nach den Fleischtöpfen Ägyptens sehnen; c'est Sodome et Gomorrhe, it's a regular Sodom, es ist ein wahres Sodom und Gomorra; sodomie, sodomy, Sodomiterei ; philistin, philistine, Philister; jouer au bon Samaritain, to be a good Samaritan, den barmherzigen Samariter spielen; arcadien, Arcadian, arkadisch; sel attique, Attic salt, attisches Salz; lesbienne, lesbian, Lesbierin; marathon, marathon (race), Marathonlauf; foi carthaginoise, Carthaginian faith, punische Treue; vandale, vandal, Vandale; Rome ne s'est pas faite en un jour, Rome was not built in a day, Rom ist nicht an einem Tage erbaut worden ; aller à Canossa, to go to Canossa, nach Kanossa gehen; il y a quelque chose de pourri dans le royaume de Danemark, something is rotten in the state of D enmark, etwas ist faul im Staate Dänemark, etc. 
La correspondance trilingue n'est pourtant pas toujours parfaite ou complète: s'endormir dans les délices de Capoue, to sink in Capuan luxury, n'a pas son équivalent toponymique en allemand; babylonien, babylonian ont le sens de gigantesque en français et en anglais, alors que l'allemand babylonisch signifie pompeux; béotien, boeotian sont orphelins de l'allemand; philistine a pris dans l'ancien argot anglais le sens d'ivrogne (xvii-xviiie s.) ; Zustände wie im alten Rom est propre à l'allemand seulement, etc.

\section{CRÉATIONS D'INSPIRATION PLUS RÉCENTE}

Parmi les termes et locutions plus récents, rares sont ceux où le même symbole se retrouve dans les trois langues: juif, Jew, Jude au sens d'usurier; lombard, lombard, Lombarde au sens de changeur ; mal français, French disease, Franzosenkrankheit pour la syphilis; mongolisme, mongolism ou mongolian idiocy, Mongolismus pour la trisomie 21 en médecine; jeunes Turcs, young Turks, Jungtürken pour désigner les jeunes d'un mouvement politique qui veulent un changement; mener une vie de bohème, to lead a Bohemian life, ein Bohemeleben führen ; mais déjà l'identité de symbole manquet-elle en allemand pour envoyer en Cornouailles, to send to Cornwall without a boat (cocufier); en file indienne, in Indian file (à la queue leu leu); aux calendes grecques, on the Greek calends (jamais) ; frères siamois, Siamese twins (au sens d'amis inséparables); châteaux en Espagne, castles in Spain (chimères); Pays-Bas, the Netherlands (argot pour le sexe de la femme), etc.

Dans la plupart des cas, les symboles diffèrent et, même ainsi, les exemples impliquant les trois langues ne sont pas légion: c'est du chinois pour moi, it's all Dutch to me, das sind mir böhmische Dörfer ; filer à l'anglaise, to take French leave, sich französisch verabschieden ; être de $\mathrm{M}$ arseille ou raconter des histoires marseillaises, to tell Texan tales (US), Geschichten aus dem Wienerwald erzählen; vendre des coquilles à ceux qui viennent de Saint-M ichel, to carry coals to Newcastle, Eulen nach Athen tragen ; capote anglaise, French letter, Pariser; envoyer quelqu'un se faire voir chez les Grecs, to send someone to Jericho, jemand nach Buxtehude wünschen; va te faire voir chez les Grecs! go to Halifax! ab nach Kassel!

Bien plus nombreux sont les cas où deux langues seulement usent d'un symbole toponymique, même différent, alors que la troisième n'en a cure. Ainsi en va-t-il du français pour les paires anglo-allemandes swede, deutsche Ananas (chou-rave); that beats the Dutch! das haut den stärksten Eskimo vom Schlitten! (c'est le comble!) ; Indian gift, I ndianergeschenk (cadeau en échange d'un autre) ; I rish grapes, preußische Beilage (pommes de terre) ; Grecian/Greek gift, ein Geschenk wie das trojanischePferd (cadeau empoisonné) ; to talk double-D utch, Kauderwelsch reden (baragouiner), etc. ; ainsi en va-t-il de l'anglais pour les paires franco-allemandes ce n'est pas le Pérou, damit wirst du nicht Amerika machen (ce n'est pas grand chose) ; œil américain, deutscher Blick (regard scrutateur); le dernier des Mohicans, der letzte Mohikaner (l'ultime exemplaire) ; mai son de Bohème, polnischeW irtschaft (grand désordre) ; fier comme un Écossais, stolz wie ein Spanier (orgueilleux, superbe), etc. ; ainsi en va-t-il de l'allemand pour les paires franco-anglaises bourguignon, Bengal blanket (argot pour soleil); canadienne de sapin, Chicago overcoat (argot pour cercueil); baiser florentin, French kiss (baiser lingual) ; breton, pedlar's French (argot du milieu); c'est l'armée bolivienne! all chiefs, no Indians! (il y a plus de chefs que d'exécutants); 
cousin à la mode de Bretagne, Kentish cousin (parent éloigné) ; palu breton, Dutchman's headache (argot pour ivresse); c'est un fin Normand, he's as cunning as a Yorkshireman (il est rusé), etc.

\section{ALLUSIONS PÉJORATIVES}

Lorsque l'imaginaire d'une langue puise ses symboles dans une représentation de l'étranger, c'est rarement pour en retenir des qualités ou des mérites (par exemple, oncle d'Amérique, le dernier des Romains, fort comme un Turc en français, Irish welcome, French curves en anglais, ein bischen schief ist englisch, alter Schwede en allemand), mais plutôt pour mettre en évidence des travers parfois réels, parfois attribués en raison d'inimitiés historiques entre peuples. C'est ainsi que le français parle de querelle d'Allemand, de soupir d'Allemand, de prendre quelqu'un pour un Allemand, de dents d'Anglaise, de filer à l'anglaise, de capote anglaise, de mal espagnol, de payer à l'espagnole, de botte florentine, d'être grec, d'envoyer quelqu'un se faire voir chez les Grecs, de faire quelque chose en juif, de mal napolitain, de boire comme un Polonais, de ventre à la Suisse, de traiter quelqu'un à la turque, etc. L'anglais parle de Chinese deal, de French disease, de to take French leave (ce qui montre que l'intention péjorative peut être réciproque), deFrench prints, de German duck, de German goiter (le ventre à la Suisse du français), de Greek fashion (la botte florentine du français), de merry as a Greek ou as drunk as a Dutchman (pour le français saoul comme un Polonais), de Dutch bargain, de Dutch courage, de Dutch treat (l'invitation à l'américaine du français), de M exican athlete, de Dutchman's headache, de Spanish coin, de Spanish gout, de Turkish treatment, etc. L'allemand parle de Pariser (pour capote anglaise), de sich französisch verabschieden (pour filer à l'anglaise), de Pappchinese, de englisch einkaufen, de Vollblutindianer, de unbeschnittener weisser Jude, de polnische Wirtschaft, de scharf wie tausend Russen, de Kümmeltürke, etc.

La péjoration peut même se pratiquer à l'intérieur d'un pays, à l'encontre des habitants d'une région particulière. Le français possède ainsi tour de Basque, être du régiment de Champagne, quelques moutons et un Champenois font cent bêtes, promesse de Gascon, s'en tirer en Gascon, tour de Gascon, être Normand, réconciliation normande, etc. ; en anglais, on trouve Cornish hug, Derbyshire born and bred, Irish evidence, to get one's Irish up, to weep Irish, Manchester bred, Scotch greys, Welsh fiddle, to put Yorkshire on someone, etc., mais aussi, pour les États-Unis, Arkansas lizard, square from Delaware, to be from Missouri, etc.

\section{INFLUENCE DE LA TOPONYMIE INDIGÈNE}

On comprend aisément qu'une langue ait tendance à se servir au premier chef des noms de localités du pays. Le français s'est ainsi forgé des expressions imagées avec Arles (jouer les Arlésiennes), Aubervilliers (chou pour chou, Aubervilliers vaut bien Paris), Cancale (envoyer à Cancale), Corbeil (prendre Paris pour Corbeil), Gravelotte (ça tombe comme à Gravelotte!), Landerneau (faire du bruit dans Landerneau), M arseille (être de Marseille), M onaco (des monacos), Nice (être niçois), Niort (aller à N iort), Paris (Paris vaut bien une messe), Pontoise (revenir de Pontoise), Rouen (aller à Rouen), Saint-M ichel (vendre des coquilles à ceux qui viennent de Saint-M ichel), 
Soissons (soissonnais), Vanves (il est sur le four de Vanves), Villejuif (mettre Villejuif dans Pontoise), etc., parfois encore avec une arrière pensée péjorative comme dans c'est une vraie forêt de Bondy, il est de Lagny, arriver comme les pompiers de N anterre.

L'anglais n'est pas en reste, qui s'empare de Bath (go to Bath !), Brighton (a bike ride to Brighton), Bristol (Bristol milk), Bungay (he's been to Bungay fair and broken both his legs), Burford (to take a Burford bait), Cambridge (Cambridge fortune), Coventry (to send someone to Coventry), Dublin (to tip someone theD ublin packet), Durham (Durham man), Halifax (go to Halifax!), Hastings (to be none of the Hastings sort), Kilkenny (to fight like Kilkenny cats), London (to put the best side to London), Manchester (M anchester-bred, long in the arms and short in the head), Newcastle (to carry coals to Newcastle), Romford (you can ride to Romford on this knife), Scarborough (Scarborough warning), Tipperary (Tipperary lawyer), Yarmouth (to go Yarmouth), etc.; les États-Unis ont fait de même avec Chicago (Chicago overcoat), M emphis (M emphis dominoes), N ew York (N ew York's finest), Philadephia (enough to puzzle a Philadelphia lawyer), etc.

L'allemand imagéa mis la main sur Berlin (Berliner), Bettenhausen (nach Bettenhausen gehen), Bonn (Bonner Volkswagen), Buxtehude (in Buxtehude wohnen), Darmstadt (Gruss aus Darmstadt), H ornberg (ausgehen wie das H ornberger Schiessen), Kassel (ab nach Kassel!), Kissingen (nach Kissingen gehen), O berammergau (O berammergauner), Pappenheim (seinePappenheimer kennen), Speyer (nach Speyer appellieren), etc.

\section{JEUX DE MOTS}

Dans plusieurs des exemples cités ci-dessus, le toponyme utilisé ne doit son choix qu'à un jeu de mots suggéré par une ressemblance de forme ou de consonance. En français, c'est le cas d'être niçois (ex. I'alsacien nix, rien), aller à Niort (ex. nier), aller à Rouen (ex. le supplice de la roue), auxquels il convient d'ajouter aller en Bavière (ex. baver), envoyer en Cornouailles (ex. corne), aller à Cracovie (ex. le populaire craque, mensonge), hambourgeois (ex. en bourgeois), prince de H ongrie (ex. hongre), japonais (ex. l'al gérois tchapes, sous), être adroit comme un prêtre normand (ex. saint Gaucher, prêtre normand), aller en Suède (ex. suer la vérole).

Le phénomène n'est pas étranger à l'anglais, qui offre to go to Bedfordshire (ex. bed, lit), to send to Cornwall without a boat (ex. l'ancien corn pour horn, corne), Gravesend bus (ex. grave, tombe et end, fin), to come from Greenland (ex. green, naif, inexpérimenté), $\mathrm{H}$ ittite (ex. to hit, frapper), gone to M oscow (ex. to mosk, mettre en gage), Needham (ex. need, besoin), to learn manners in Seville (ex. civil), to come from Tripoli (ex. to trip, trébucher), to be gone to the diet of Worms (ex. worms, vers). Parfois, seuls une prononciation approximative ou un télescopage fantaisiste expliquent les toponymes retenus, tels Bucklebury (pour buggery, pédérastie) ou Gomorrah to you! (pour good morning to you! bonjour à vous).

Parmi les exemples allemands obtenus par le même procédé, il y a nach Bettenhausen gehen (ex. Bett, lit), Cassablanca (ex. Kasse, argent et blank, sans le sou), Gruss aus D armstadt (ex. Darm, intestin), nach Kissingen gehen (ex. Kissen, oreiller), noblenz Koblenz (pour le latin nolens volens), 0 berammergauner (ex. Gauner, escroc), nach Speyer appellieren (ex. speien, cracher). Cette propension au jeu de mots peut 
même conduire à la création de toponymes fictifs, comme Dummsdorf (aus Dummsdorf sein, être de son village), Kleinkleckersdorf (patelin, bled), Nackedonien (camp nudiste, par attraction de M azedonien), auxquels feraient pendant le français Fouilly-les-Oies (habiter à Fouilly-les-Oies) et l'anglo-américain Hicksville (to live in Hicksville).

\section{CRÉATIONS AU SECOND DEGRÉ}

Le lien entre le toponyme choisi et le sens attribué à l'image qu'il contribue à former n'est pas toujours évident au premier abord et nécessite dès lors le passage par un stade d'interprétation préalable.

Si l'argot français nomme francforts les doigts de la main, c'est en raison d'une ressemblance aux saucisses de Francfort; s'il traduit la mauvaise haleine par «avoir les dents plombées au Gorgonzola», c'est par allusion à l'odeur du fromage de Gorgonzola; si «morceau de gruyère» décrit un visage marqué par la petite vérole, c'est en référence aux trous du fromage en provenance de Gruyère. Si «la macédoine de fruits» du langage courant désigne un mélange de fruits coupés en morceaux, c'est pour rappeler l'empire d'Alexandre composé d'éléments divers; si maroquin désigne un portefeuille ministériel et si anciennement «péter le maroquin à quelqu'un » signifiait infliger une correction, c'est en souvenir d'un cuir dont le $M$ aroc a été le premier lieu de production; si, en médecine, le terme impropre de mongolisme est synonyme de syndrome de D own, c'est parce que le patient atteint de cette maladie, appelé aussi mongolien, accuse des traits semblables à ceux de la race mongole; si «bon comme la romaine» qualifie une bonté à toute épreuve, c'est en mémoire de la laitue romaine importée d'Italie au xve siècle; si, au xvii siècle, le français a pu dire «aller en Suède» pour se fair re traiter de la syphilis, c'est parce que les vertus supposées de sudorifiques ont permis le jeu de mots avec le verbe suer.

De même, en anglais, «Chinese landing» ne peut se comprendre qu'en passant par one wing low écrit à la chinoise Wun Wing Lo (!) ; pour les américanismes «china chin » et «china clipper», il faut se souvenir que china signifie porcelaine; Dalmatian pudding repose sur l'analogie entre l'aspect des raisins secs et les taches noires de la robe du chien dalmatien; Gorgonzola ! est une allusion à l'excellence du fromage du même nom ; «to make hamburger of someone» se réfère au bifteck haché servi dans un petit pain sous le nom de hamburger; si anciennement le pilori se disait «N orway neck-cloth », c'est parce qu'il était souvent fait de sapin de Norvège; russia renvoie au cuir (Russian leather) dont est fait le portefeuille; «to set the swede down » ne devient intelligible qu'après le rapprochement entre swede et tête; «Old Brown Windsor» provient de la couleur d'un savon ainsi nommé; etc.

L'allemand aussi possède quelques créations de ce type, telles «chinesischer Spinat» qui interprète une graphie chinoise fantaisiste de Kuhkacke (Ku-Ka-Ke!), Ithaker qui ne concerne en rien I'antique Ithaque mais serait issu d'un télescopage entre I taliener et Italiker (peuplade de I'Italie ancienne), Korinthenkackerei qui résulte de la filiation Korinthen $=$ raisins secs $=$ crottes de mouton . 


\section{FANTAISIES ARGOTIQUES}

L'anglais non conventionnel se singularise par un procédé de rhyming slang (argot rimé, RS) qui consiste à créer des vocables nouveaux en remplaçant le terme d'origine par un ou plusieurs autres, choisis arbitrairement mais rimant avec lui. Les noms de pays, de nationalités, de régions et de localités sont fréquemment appelés à remplir cette fonction: Abergavenny et Kilkennny (pour penny), Berkeley Hunt et Berkshire Hunt (pour cunt), Burton-on-Trent (pour rent), Chatham and Dover (pour to give over), Cheltenham bold (pour cold), China plate (pour mate), Dublin tricks (pour bricks), Egyptian Hall (pour ball), Epsom races (pour braces ou faces), Everton toffee (pour coffee), France and Spain (pour rain), South of France (pour dance), German bands (pour hands), German flutes (pour boots), Hampstead Heath (pour teeth), Lakes of Killarney (pour crazy), Lancashire lass (pour glass), London fog (pour dog), Maidstone jailer (pour tailor), Dutch ou Scotch pegs (pour legs), New York nippers (pour kippers), Oxford scholar (pour collar ou dollar), gates of Rome (pour home), Russian duck (pour muck), Russian Turk (pour work), Scotch peg (pour egg), Spanish guitar (pour cigar), Sidney barber (pour harbour), Warwick Farms (pour arms), West Ham reserves (pour nerves), Yarmouth bloater (pour motor car), Duke of York (pour to walk, to talk, cork ou chalk), Duke of Yorks (pour forks).

II arrive que le mot choisi pour la rime soit lui-même déjà un mot d'argot, comme dans Hampton Wick (pour prick) et Yarmouth tike (pour mike) ; il arrive aussi que le mot de la rime disparaisse à l'usage, comme dans berkeley (pour Berkeley Hunt), burton (pour Burton-on-Trent), china (pour China plate), dukes (pour Duke of Yorks, dans le sens de mains dérivé de forks), old Jamaica (pour old Jamaica rum, assonance plutôt que rime de sun).

\section{LE CORPUS}

Les termes et expressions sont tout d'abord définis, puis rendus dans les deux autres langues si possible par un équivalent de la même symbolique, sinon par un autre tour imagé ou, à défaut, par une traduction simplement explicative.

Les entrées du corpus ont été puisées tant dans le passé que dans le présent. On y trouvera donc des tournures anciennes, certaines toujours usitées, d'autres obsolètes, à côté de créations contemporaines. Mais à quel moment un vocable, une locution deviennent-ils obsolètes? Le proverbe Rome ne s'est pas faite en un jour, bien que datant du $x v{ }^{e}$ siècle, est toujours en usage; récemment encore, un commentateur de télévision s'exclamait sous une pluie battante: ça tombe comme à Gravelotte! expression née après la guerre franco-allemande de 1870. Les seuls dont on puisse dire avec certitude qu'ils sont désuets sont ceux qui se rapportent à des objets ou pratiques disparus: le bourreau (M onsieur de Paris, en français), le traitement de la syphilis (aller en Suède, en français encore), le pilori (Norway neck-cloth ou Scotch casement, en anglais), la pendaison (salade de Gascogne en français, Bridport dagger ou California collar en anglais), etc. Nous nous bornerons donc à indiquer, chaque fois que faire se peut, la date d'apparition approximative de chaque entrée en laissant au lecteur le soin d'apprécier leur éventuelle désuétude en fonction de sa culture linguistique propre. 
Le corpus s'est nourri aussi à ce que l'on nomme communément les différents niveaux de langage. Mais il serait sans doute malvenu de vouloir appliquer ici ces repères classiquement adoptés dans les dictionnaires. Quand une expression est-elle familière, populaire, argotique, vulgaire? Les échelles de valeurs varient selon les époques et les individus. De plus, une telle classification en fonction de niveaux sociaux est très aléatoire du fait que le jugement est porté par le représentant d'un niveau particulier. Aussi laisserons-nous le lecteur faire la part des choses d'après ses habitudes langagières personnelles et seules les entrées récusées par la langue acadé mique seront-elles suivies du signe NC (non conventionnel').

\section{NOTE}

1. Dans le sens défini, d'une part, par J. Cellard et A. Rey (1991) et, d'autre part, par E. Partridge (1961).

\section{SÉLECTION BIBLIOGRAPHIQUE}

Cel I ard, J. et A. Rey (1991) : Dictionnaire du français non conventionnel, Paris, Hachette.

Col in, J.-P. et J.-P. M ével (1992) : Dictionnaire de l'argot, Paris, Larousse.

D eak, E. (1962) : Grand dictionnaire d'américanismes, Paris, Éditions du Dauphin.

De Dony, Y. (1951) : Léxico del Lenguaje figurado, Buenos Aires, Desclée de Brouwer.

Dun et on, C. et S. Cl aval (1990) : Le bouquet des expressions imagées, Paris, Seuil.

Fr iedr ich, W. (1960) : M oderne deutsche Idiomatik, M ünchen, Hueber.

Küpper , H. (1955-70) : W örterbuch der deutschen Umgangssprache (6 Bde), H amburg, Claasses.

Leit ner, M. J. and J. R. Lanen (1965): Dictionary of French and American Slang, New York, Crown Publishers.

M ansion, J. E. (1980) : H arrap's Standard French-English Dictionary (4 vol.) , London, G. Harrap \& $C^{\circ}$.

Marks, J. (1971) : Harrap's French-English Dictionary of Slang and Colloquialisms, London, G. Harrap \& $\mathrm{C}^{\circ}$.

M essinger , H. (1959) : Langenscheidts Handwörterbuch Deutsch-English, Berlin, Langenscheidt.

M ur et , E., D. Sander s und O. Spr inger (1962) : Langenscheidts Enzyklopädisches Wörterbuch English-Deutsch (2 Bde), Berlin, Langenscheidt.

Onions, C. T. (1969) : The Shorter Oxford English Dictionary (2 vol.), Oxford, Clarendon Press.

Partridge, E. (1961): A Dictionary of Slang and Unconventional English (2 vol.), London, Routledge \& Kegan Paul.

Pfohl, E. und P. Benoît (1969) : Brockhaus-Bilderwörterbuch Französisch-Deutsch, Wiesbaden, Brockhaus.

Phyt hian, B. A. (1986) : A Concise Dictionary of English Slang, London, Hodder \& Stoughton.

Taylor, R. and W. Gottschalk (1960): A German-English Dictionary of Idioms, München, Hueber. 


\section{LEXIQUE D'EXPRESSIONS FRANÇAISES}

\section{ALLEMAGNe}

c'est toujours ça que les Allemands n'auront pas! $\left(20^{\mathrm{e}}\right)$

allusion aux réquisitions sous l'occupation; se dit de qqch que l'on a pu soustraire (au fisc, etc.)

(E) said about sthg one has been able to preserve or take away

(D) wird gesagt von etwas, dass man hat bewahren oder entziehen können

c'est de l'allemand/du haut-allemand pour moi ! (17)

1. c'est un charabia inintelligible 2. c'est un sujet auquel je n'entends rien

(E) it's all Dutch/Greek to me!

(D) das sind mir böhmische/spanische

Dörfer!, das ist chinesisch für mich

contrefaire l'Allemand (17 $)$

feindre d'être grossier

(E) to pretend to be rude

(D) sich grob stellen

Dieu nous garde de la santé des Allemands et de la maladie des Français (17 ${ }^{\mathrm{e}}$ )

qu'll nous garde de trop boire et d'avoir la syphilis

(E) God save us from drinking and from the pox

(D) Gott bewahre uns vor übermässigem

Trinken und vor der Syphilis

faire une querelle d'Allemand à qqn (17 ${ }^{\mathrm{e}}$

chercher querelle sans motif

(E) to pick a forced quarrel with

(D) einen Streit vom Zaune brechen

flûte d'Allemand (17e)

grand verre

(E) a large glass

(D) ein grosses Glas

gare à la queue des Allemands! (1842)

attention aux suites fâcheuses d'une affaire

(E) look out for the untoward consequences of a business

(D) Vorsicht für die ärgerlichen Folgen eines Geschäfts

peigne de l'Allemand (17e)

la main

(E) Duke of Yorks (NC, 1874)

(D) Flosse (NC, 1870)

prendre qqn pour un Allemand (17 ${ }^{\mathrm{e}}$

le prendre pour un niais

(E) to take s.o. for a simpleton

(D) jn für einen Dummkopf halten querelle d'Allemand (17e)

fondée sur peu ou sur rien

(E) trumped-up quarrel

(D) Streit um des Kaisers Bart

saut de l'Allemand (17e)

qui mène du lit à la table

(E) from the bed to the table

(D) Sprung vom Bett zum Tisch

soupir d'Allemand (17e)

éructation

(E) burp (NC, 1923)

(D) Bäuerchen (NC, 1900)

\section{ALLOBROGES}

c'est un Allobroge! (1842)

1. individu stupide, ignorant

2. grossier personnage

(E) 1. Goth (1663) 2. boar

(D) 1. Doofkopf (NC, 20e) 2. grober Klotz

(NC, 18)

AMÉRIQUE

oncle d'Amérique (1826)

oncle fortuné

(E) rich uncle

(D) reicher Onkel in Amerika

avoir l'œil américain (1862)

1. voir tout du premier coup d'œil

2. surveiller

(E) 1. to keep one's eyes skinned 2. to keep an eye on

(D) 1. ein scharfes Auge haben 2. ein wachsames Auge haben

invitation à l'américaine

où chacun apporte son écot

(E) Chinaman's shout (NC, 20), Dutch treat

(NC, 1875)

(D) amerikanische Einladung

œil américain (1872)

1. regard scrutateur 2. regard séducteur

(E) 1. searching look 2. flirtitious look

(D) 1. deutscher Blick (NC, 1933)

2. flirtender Blick

vol à l'américaine (1862)

vol doublé d'une mystification

(E) confidence trick

(D) frech angelegter Diebstahl

\section{ANGLETERRE}

avoir les/ses Anglais (NC, 1867)

avoir ses règles

(E) to have the monthlies (NC, 1857)

(D) d.u. sein (NC) 
capote anglaise (NC, 1878)

préservatif masculin

(E) American (NC, 20)/French (NC, 1870)/

Italian (NC, 18e-20)/Spanish (NC, 18-20

letter, Port Said garter (NC, 20")

(D) Pariser (NC, 19e)

dents d'Anglaise (20)

dents proéminentes

(E) projecting teeth

(D) vorspringende Zähne

faire la semaine anglaise (1911)

avoir ses fins de semaine libres

(E) to have one's week-ends free

(D) die englische Arbeitszeit haben

faire une anglaise (NC, 1867)

payer sa part chacun

(E) to eat in Dutch street (NC, 19e-20 $)$, to

go Dutch (NC, 1914)/Yorkshire (19e-20e)

(D) jeder für sich bezahlen

filer à l'anglaise (1867)

partir subrepticement

(E) to take Dutch (US)/French leave (NC,

1770)

(D) sich französisch verabschieden

il y a des Anglais dans cette rue là! (17 $)$

se disait d'une rue à éviter pour cause de dettes

(E) said of a street to be avoided because of debts

(D) gesagt von einer Strasse, die man wegen Schulden vermeiden muss

jouer à l'anglaise (1867)

jouer pour de l'argent

(E) to play for money

(D) für Geld spielen

jouer au billard anglais (NC, 1991)

pratiquer l'acte sexuel

(E) to fuck (NC, 1800)

(D) bimsen (NC, 1900)

les Anglais ont débarqué (NC, 1832)

(cf. avoir ses AN GLAIS)

lieux à l'anglaise (1856)

le water-closet

(E) the john (NC, 20)

(D) das Klo (NC, 20)

mariage à l'anglaise (19e)

où chacun vit de son côté

(E) marriage without cohabitation

(D) Heirat ohne Zusammenleben

momignardage à l'anglaise (NC, 1878)

fausse couche

(E) miscarriage

(D) Fehlgeburt pisser à l'anglaise (NC, 1867)

partir au moment décisif

(E) to leave at the critical moment

(D) im entscheidenden Moment verschwinden

semaine anglaise (1911)

semaine de travail de $5 \frac{1}{2} 2$ jours

(E) week of $5 \frac{1}{2}$ working days

(D) englische Arbeitszeit

\section{APACHES}

apache (NC, 1902)

bandit parisien, rôdeur de grande ville

(E) tough (NC, 1910)

(D) Rowdy (NC, 1855)

\section{ARABIE}

arabe (17)

1. usurier 2. homme dur en affaires

(E) 1. Jew (1845) 2. Jew

(D) 1. Jude 2. Jude

bureau arabe (NC, 1872)

mélange d'absinthe et d'orgeat

(E) mixture of absinthe and orgeat syrup

(D) M ischung von Wermut und Mandelmilch

combines arabes (19e)

moyens astucieux, micmac

(E) tricky business, scheming

(D) tückische Tricks

fourbi arabe (NC, 1914)

(cf. ci-dessus)

liqueur arabe

café

(E) coffee

(D) Kaffee

téléphone arabe $\left(20^{\circ}\right)$

le bouche à oreille

(E) grapevine (telegraph) (NC, 1920)

(D) Flüsterpropaganda (NC)

\section{ARCADIE}

rossignol/roussin d'Arcadie (1842)

1. âne 2. cf. ALLOBROGE 1

(E) 1. Jerusalem pony 2. cf. ALLOBROGE 1

(D) 1. Ia (NC, 19e) 2. cf. ALLOBROGE 1

arcadien

d'une simplicité pastorale

(E) Arcadian

(D) arkadisch

\section{ARLES}

jouer les Arlésiennes (20

être célèbre sans jamais se montrer

(E) to be famous without going public

(D) berühmt sein ohne an die Öffentlichkeit zu treten 


\section{ARMÉNIE}

ça ne sent pas le papier d'Arménie!

ça pue!

(E) it doesn't smell of attar of roses!

(D) es riecht wie ein Veilchen! (NC, 1930)

\section{ATHÈNES}

Athénien

personne d'un goût délicat et d'une culture raffinée

(E) a person of refinement and culture

(D) ein Mensch feiner Geschmack und Kultur

c'est ici que les Athéniens s'empoignèrent! $\left(20^{\circ}\right)$ voilà où est la difficulté

(E) there's the rub! (16)

(D) da liegt der Hund begraben! (NC, 18e)

\section{ATTIQUE}

foi attique

fidélité à toute épreuve

(E) Attic faith

(D) ewige Treue

sel attique

esprit caustique

(E) Attic salt

(D) attisches Salz

\section{AUBERVILLIERS}

chou pour chou, Aubervilliers vaut bien Paris

(17)

c'est tout un, c'est pareil

(E) it's much of a muchness

(D) das ist einerlei

\section{AUVERGNE}

cure-dent d'Auvergne (NC, 17)

le membre viril

(E) Hampton wick (NC, 19e-20e) Irish root

(NC, 1830-1914)

(D) Ding (NC, 16)

Auvergnat (NC, 1977)

1. Juif 2. charabia

(E) 1. Jew 2. double-Dutch (NC, 1860), Greek (1600)

(D) 1. Jude 2. Kauderwelsch

avaler l'Auvergnat (NC, 1881)

communier

(E) to receive Holy Communion

(D) das Abdendmahl empfangen

les Auvergnats et les Limousins font leurs

affaires, puis celles de leurs voisins (1618)

les Auvergnats s'occupent de leurs intérêts en premier

(E) the natives of Auvergne act in their own interest first

(D) die Bewohner der Auvergne kümmern sich erst um ihr eigenes Interesse

\section{AZTÈQUES}

Aztèque (1861)

avorton

(E) a shrimp of a man

(D) Knirps

\section{BABYLONE}

babylonien (16e)

gigantesque

(E) Babylonian (16)

(D) riesenhaft

\section{BASQUES}

aller à pied comme un Basque (17)

marcher vite et longtemps

(E) to walk quickly and for a long time

(D) lang und im schnellen Tritt gehen

courir comme un Basque (17e)

courir très vite

(E) to run like a hare

(D) sehr schnell laufen

les Basques disent qu'ils se comprennent, mais $c^{\prime}$ 'est un mensonge (16e)

allusion à la difficulté de la langue basquaise

(E) an allusion to the difficulty of the

Basque language

(D) Anspielung auf die Schwierigkeit der baskischen Sprache

le tour du Basque (1842)

croc-en-jambe

(E) leg-trip

(D) Beinstellen

parler français comme un Basque l'espagnol

(17)

parler fort mal le français

(E) to speak French very poorly

(D) das Französische radebrechen

un tour de Basque (17e)

supercherie

(E) deceit, piece of trickery

(D) hinterlistiger Streich

\section{BAS-RHIN}

le (département du) Bas-Rhin (NC, 19e) le derrière

(E) Jutland (NC, 18 $\left.-19^{\mathrm{e}}\right)$, Khyber Pass (NC,

19-20'), Westphalia (NC, 1904)

(D) Podex (NC, 17)

\section{BAVIÈRE}

aller en Bavière (17)

contracter la syphilis

(E) to contract syphilis

(D) mit Syphilis angesteckt werden

bavarois (NC, 1916)

pou

(E) Arkansas lizard (US), Scotch grey (NC,

19e-20), Scotchmen (NC, 1887)

(D) Biene (NC, 1870) 


\section{BEAUCE}

plume de Beauce (19e)

paille

(E) straw

(D) Stroh (halm)

\section{BÉDOUINS}

Bédouin

1. homme brutal et inculte (19e) 2. crucifix

(NC, 20)

(E) 1. savage, rough fellow 2 . crucifix

(D) 1. wüster, ungebildeter Mensch

2. Kruzifix

\section{BELGIQUE}

fume, c'est du belge! (NC, 1977)

apostrophe de refus grossièrement injurieuse

(E) go and get fucked! (NC, 19e-20)

(D) leck mich am Arsch! (NC, 18)

filer en Belgique (19)

lever le pied (financier, banquier, etc.)

(E) to bolt (financier, banker, etc.)

(D) durchbrennen

\section{BELLEVILLE}

pucelle de Belleville (1864)

prostituée

(E) Broadway broad (US, NC, 1930), Covent

Garden nun, Dutch widow (16 $\left.-18^{\mathrm{e}}\right)$

(D) Prostituierte

\section{BÉOTIE}

Béotien (1831)

1. cf. ALLOBROGE 12 . profane

(E) 1. Bœotian 2. Philistine

(D) 1. cf. ALLOBROGE 12 . Philister

\section{BERRY}

c'est un mouton du Berry, il est marqué sur le nez (17)

il a une balafre sur le nez

(E) his nose is scarred

(D) seine Nase zeigt eine Schmarre

\section{BOHÈME}

la bohème (1842)

vie précaire, au jour le jour

(E) Bohemia (of the artistic world)

(D) Boheme (wesen)

mener une vie de bohème, vivre comme un

bohème (1842)

vivre sans ordre ni règle

(E) to lead a Bohemian life

(D) ein Bohemeleben führen

bohémien (1467)

tzigane

(E) Bohemian (1696), Egyptian (16e)

(D) Zigeuner

\section{BOLIVIE}

c'est l'armée bolivienne! (20)

il y a plus de chefs que d'exécutants
(E) all chiefs, no Indians!

(D) hier ist jederman Chef !

\section{BONDY}

c'est la forêt de Bondy ! c'est une vraie forêt de Bondy! (18-19e)

c'est du vol manifeste, on m'a estampé

(E) that's a Dutch reckoning! it's a regular

Hounslow Heath!

(D) das ist eine richtige Erpessung!

\section{BOURGOGNE}

bourgogne

nez d'ivrogne

(E) geranium (NC, 1882)

(D) Tulpe (NC, 1850)

bourguignon (NC, 1821)

le soleil

(E) Bengal blanket (NC, 19e-20 $)$, old Jamaica

(NC, 19e-20), Spanish faggot (NC, 1785-

1850)

(D) Schweissbrenner (NC, 1960)

jurer comme un Bourguignon (1842)

lâcher juron sur juron

(E) to swear like a trooper

(D) fluchen wie ein Fuhrknecht

\section{BRÉSIL}

brésilienne (NC, 1884)

travesti

(E) transvestite

(D) Transvestit

\section{BRETAGNE}

parent à la mode de Bretagne (17e)

de parenté (très) éloignée

(E) Kentish cousin

(D) weitläufiger Verwandter breton

1. témoin de duelliste (Moyen Âge) 2. argot

(NC, 1795)

(E) 1. duellist's second (M iddle Ages)

2. pedlar's French

(D) 1. Zweikämpfersekundant (M ittelalter)

2. Gaunersprache

c'est du bas-breton pour moi ! (1790)

(cf. c'est de l'ALLEM AND!)

coup de tête de Breton

coup de tête dans la poitrine

(E) butt in the stomach

(D) Kopfstoss in die Brust

saut de Breton (17)

(cf. tour du BASQUE)

\section{BRIE}

la barbe lui croît avant l'entendement, comme aux chèvres de Brie (17e)

la barbe vient avant l'expérience

(E) a beard grows before experience

(D) der Bart kommt vor die Erfahrung 


\section{BRUXELLES}

filer à Bruxelles

(cf. filer en BELGIQUE)

\section{BULGARIE}

pissenlit bulgare (NC, 1984)

position de coït

(E) a love making posture

(D) eine Stellung beim Geschlechtsverkehr

\section{BYZANCE}

C'est Byzance! (1965)

c'est l'abondance, c'est magnifique

(E) it's like the garden of Eden!

(D) das ist ein richtiges Schlaraffenland!

discussions/querelles byzantines (19)

discussions futiles et oiseuses

(E) futile and pointless discussions

(D) spitzfindige, eitele Auseinandersetzungen

\section{CACHEMIRE}

cachemire (NC, 1866)

torchon

(E) duster, kitchen cloth

(D) Abwischtuch

\section{CAEN}

poitrine à la mode de Caen $\left(20^{\circ}\right)$

seins flasques

(E) drooping breasts

(D) schlaffer Busen

\section{CANADA}

canadas

pommes de terre

(E) Irish apples (NC, 19e)/apricots (NC, 1815)/grapes (US, 19e)/lemons (NC, 1815), M unster plums (NC, 1780, ob.)

(D) preussische Beilage (NC, 20)

canadienne en sapin (NC, 20)

cercueil

(E) Chicago overcoat (US, NC, 1929)

(D) Sarg

\section{CANCALE}

envoyer à Cancale (17e)

chasser rudement

(E) to send to Bath (NC, 1830)/to Jericho

(NC, 17e)

(D) nach Buxtehude wünschen (NC, 19e)

\section{CANOSSA}

aller à Canossa (19e)

s'incliner honteusement, s'humilier

(E) to go to Canossa (19e)

(D) nach Kanossa gehen, den Kanossaweg antreten (19e)

\section{CAPHARNAÜM}

capharnaüm (1833)

1. amas d'objets en désordre 2 . lieu renfermant ces objets
(E) 1. a regular jumble 2. gloryhole

(D) 1. polnische Wirtschaft (NC, 1835) 2.

Rumpelkammer

\section{CAPOUE}

les délices de Capoue

oisiveté voluptueuse

(E) voluptuous idleness

(D) wohllustiger M üssiggang

s'endormir dans les délices de Capoue

perdre un temps précieux que l'on pourrait

employer avantageusement

(E) to sink in Capuan luxury

(D) Zeit vergeuden, die man recht gut benutzen könnte

\section{CARTHAGE}

foi carthaginoise

traitrise

(E) Carthaginian/Punic faith

(D) punische Treue

\section{CAYENNE}

cayenne (NC, 19)

1. cimetière 2 . coin perdu

(E) 1. cemetery 2. off-the map place

(D) 1. Kirchhof 2. Kleinkleckersdorf (NC, 1920)

\section{CHAM PAGNE}

être du régiment de Champagne (1842)

se moquer de l'ordre

(E) not to care for orderliness

(D) für Ordnung nicht viel übrig haben

il ne sait pas toutes les foires de Champagne (17 $)$

il ignore beaucoup de choses qui se trouvent à son préjudice

(E) he is ignorant of many things detrimental to him

(D) er ist in vielen Sachen unwissend, die für inn schädlich sind

regarder en Gâtinais/Picardie pour voir si la

Champagne brûle (1842)

loucher

(E) to be looking seven ways for Sunday

(NC, 19e-20)

(D) mit dem linken Auge in die rechten

Westentasche sehen (NC, 19e)

quelques moutons et un Champenois font cent bêtes

les Champenois sont réputés sots

(E) the natives of Champagne are reputedly stupid

(D) die Einwohner der Champagne gelten für dumm

\section{CHINE}

chinois

1. individu bizarre, original (1820) 
2. cf. cure dent d'AUVERGNE (NC, 1920)

3. compliqué, difficile (adj.)

(E) 1. a queer fish (NC, 19e-20e) 2. cf. curedent d'AUVERGNE 3. complicated; pernickety

(D) 1. komischer Kauz 2. cf. curedent

d'AUVERGNE 3. kompliziert, heikel

brouette chinoise (NC, 18)

position de coït

(E) a love-making posture

(D) eine Stellung beim Geschlechtsverkehr

ça ne fait pas mes chinois! (NC)

ça ne fait pas mon affaire

(E) that doesn't suit my book !

(D) damit bin ich nicht geholfen!

c'est du chinois pour moi ! (1790)

(cf. c'est de l'ALLEM AND!)

faire le chinois

faire des difficultés, chercher des complications

(E) to be pernickety

(D) heikel sein

prendre le Chinois par la natte (NC, 1984)

1. abuser de digressions 2 . perdre du temps inutilement

(E) 1. to lose oneself in digressions 2. to waste time to no purpose

(D) 1. sich in Abschweifungen stürzen 2. seine Zeit vergeuden

se battre/polir le chinois (NC, 1920)

se masturber

(E) to jerk off (NC, 18-20e)

(D) pimmeln (NC, 1900)

supplice chinois $\left(20^{\circ}\right)$

tourment sans nom

(E) anguish, torment beyond words

(D) ungeheuer Qual

tremper ses chinois (NC, 20)

s'impliquer

(E) to get involved

(D) sich auf etwas einlassen

chinoiseries (1845)

complications inutiles

(E) unnecessary complications, fuss

(D) übertriebene Komplikationen

\section{CORBEIL}

prendre Paris pour Corbeil (17e)

commettre une bévue, se méprendre

(E) to pull a boner (NC, 20)

(D) auf dem falschen Dampfer sitzen (NC, 1930)

\section{CORINTHE}

tout le monde ne peut pas aller à Corinthe faute de moyens, il faut renoncer
(E) failing resources, one has to give up

(D) ohne Mittel muss man aufgeben

\section{CORNOUAILLES}

aller en Cornouailles (17e)

être fait cocu

(E) to go round Cape Horn (NC, 18-19e)

(D) zum Hahnrei gemacht werden

chevalier de Cornouailles (17e)

cocu

(E) cuckold

(D) Hahnrei

envoyer en Cornouailles (17)

cocufier (son mari)

(E) to send to Cornwall without a boat (NC,

1565-1830)

(D) zum Hahnrei machen

\section{CRACOVIE}

aller à Cracovie (1842)

mentir

(E) to fib (NC, 18')

(D) kohlen (NC, 18)

avoir ses lettres de Cracovie (1842)

être un menteur avéré

(E) to be an established liar

(D) ein bezeugter Lügner sein

\section{DAHOMEY}

dahoméen (NC, 1912)

l'anus

(E) Old Brown Windsor (NC, 1942)

(D) Auspuff (NC, 1914-18)

\section{DAMAS}

trouver son chemin de Damas

changer subitement d'idées ou de convic-

tions

(E) to see the light

(D) seinen Tag von Damaskus erleben

\section{DANEMARK}

il y a quelque chose de pourri dans le royaume de Danemark (17e)

il y a qqch qui cloche dans cette affaire

(E) something is rotten in the state of

Denmark (17e)

(D) etwas ist faul im Staate Dänemark

(1850)

\section{ÉCOSSE}

percé à jour comme un poignard d'Écosse (17e)

plein de trous (d'usure)

(E) full of holes (through wear)

(D) (vor Abnutzung) durchlöchert

douche écossaise (1930)

1. désillusion 2. anicroche

(E) 1. disappointment 2. hitch

(D) 1. Enttäuschung 2. Haken 
en Écossais (1872)

en chemise

(E) without trousers

(D) ohne Hose

fier comme un Écossais (17e)

hautain et superbe

(E) haughty and vainglorious

(D) hochmütig und prahlerisch

hospitalité écossaise (19e)

bon accueil, hospitalité désintéressée

(E) hearty welcome, free hospitality

(D) herzliches Willkommen, freie

Gastfreundschaft

remède écossais ( $\left.16^{\circ}\right)$

préservatif et curatif de la syphilis

(E) prophylactic and remedy against syphilis

(D) Schutz- und Heilmittel gegen Syphilis

\section{ÉGYPTE}

blond d'Égypte (17)

Maure, Noir

(E) Moor, Negro

(D) Maure, Neger

gardez-vous de retourner en Égypte!

ne retournez pas aux vanités du monde

(E) do not go back to the futility of worldly pleasures

(D) lassen Sie es sein mit den irdischen Vergnügen

regretter les oignons d'Égypte

regretter une situation antérieure

(E) to sigh for the fleshpots of Egypt

(D) sich nach den Fleischtöpfen Ägyptens sehnen

\section{ÉPINAL}

ça fait image d'Épinal

c'est plutôt naïf (de style)

(E) it's rather naive (in style)

(D) das ist ziemlich naiv (im Stil)

\section{ESPAGNE}

bâtir/faire des châteaux en Espagne (13e)

faire des projets chimériques

(E) to build castles in Spain (19e-20e)

(D) Luftschlösser bauen

auberge espagnole (19e)

lieu où l'on ne trouve que ce qu'on y

apporte

(E) a place where you find only what you

bring with you

(D) ein Ort wo man nur das findet was man

mit sich bringt

avoir le ventre à l'espagnole

avoir le ventre vide, avoir faim

(E) too feel peckish (NC, 18e-20)

(D) Kohldampf schieben (NC, 19) couleur Espagnol malade

nuance verdâtre

(E) a sickly greenish colour

(D) ekelhafte grüne Färbung

le feu des Espagnols (17e)

(cf. BOURGUIGNON)

mal espagnol (15e)

la syphilis

(E) French ache (17-19e), Neapolitan disease

(NC, 16e-17e), Spanish gout (17e-19e)

(D) Franzosenkrankheit

marcher à l'espagnole (17e)

d'un pas solennel, gravement

(E) to walk in a solemn gait

(D) eine ernsthafte, prunkvolle Gangart

haben

parler français comme une vache espagnole (17e)

(cf. parler français comme un BASQUE

l'espagnol)

payer à l'espagnole (17e)

payer de coups ou de menaces

(E) to pay with blows or threats

(D) mit Prügel oder Drohungen bezahlen

se chauffer à l'espagnole (17)

se chauffer au soleil

(E) to warm oneself in the sun

(D) sich in der Sonne wärmen

\section{ESQUIMAUX}

esquimau (NC, 20)

chocolat glacé

(E) Eskimo pie (NC, 20)

(D) kalter Kuss (NC, 20)

\section{FLORENCE}

baiser florentin

baiser passionné sur la bouche

(E) French kiss (NC, 1923)

(D) Fünfminutenbrenner (NC, 19)

botte florentine (1789)

coït anal

(E) sodomy (13e-14e), Bucklebury (NC, 1923), Greek love/way (US, NC, 20)), Italian manner (NC, 19e)

(D) Sodomiterei

\section{FRANCE}

carte de France

taches de sperme sur les draps

(E) map of England// reland (NC, 19e-20e)

(D) Samenflecken auf dem Betttuch

Dieu nous garde de la santé des Allemands et de la maladie des Français

(cf. ALLEM AGNE)

en bon français (17)

clairement, sans ménagement

(E) in plain English (17)

(D) auf gut Deutsch (16e) 
entendre le français (17)

comprendre un avertissement, une menace

(E) to understand a warning, a threat

(D) eine M ahnung, eine Drohung verstehen

être fou en français et en latin (17e)

citer mal à propos du français et du latin

(E) to make untimely quotations in French

and Latin

(D) unpassende Zitate auf französisch und lateinisch machen

impossible n'est pas français

rien n'est impossible

(E) nothing is impossible

(D) nichts ist unmöglich

mal français

(cf. mal ESPAGNOL)

marguerites françaises (17)

compliments

(E) complimentary remarks

(D) Komplimente

parler français (17e)

s'exprimer franchement

(E) to use plain English (17e)

(D) (alt)deutsch reden

parler français comme une vache espagnole

(cf. parler français comme un BASQUE l'espagnol)

si le diable sortait de l'enfer pour se battre, il se présenterait aussitôt un Français pour accepter le défi (1842)

dicton qui vante le courage français

(E) a saying that praises French courage

(D) ein Sprichwort zum Lob des französischen Mutes

tu ne comprends plus le français?

tu ne veux pas écouter?

(E) don't you understand plain English?

(D) du verstehst wohl kein Deutsch mehr/ wohl nicht mehr Deutsch!

\section{FRANCFORT}

francforts (NC, 1982)

les doigts

(E) Duke of Yorks (NC, 1874)

(D) Griffel (NC, 19e)

\section{GASCOGNE}

salade de Gascogne (17e)

corde de chanvre (pour pendaison)

(E) Bridport dagger (NC, 17e-19e), California

collar (US)

(D) Strang

Gascon (17e)

1. fanfaron, vantard 2. railleur

(E) 1. Spanish athlete (US, NC, 1880)

\section{2. scoffer}

(D) 1. Dicktuer (NC, 1800) 2. Spottdrossel

(NC, 1850)

faire quelque chose en Gascon (17)

procéder par un tour habile

(E) to achieve sthy by cunning

(D) etwas listigerweise erreichen

faire le Gascon (17e)

se vanter

(E) to talk big (NC, 19e)

(D) gross tun (NC,17)

histoires de Gascon (17)

histoires exagérées

(E) Texan tales (US)

(D) kalter Aufschnitt (NC, 20')

il ressemble le Gascon : il n'a qu'un vice, il est trop vaillant (17)

c'est un poltron

(E) he's a quitter (NC, 20)

(D) er ist ein Hasenfuss (NC, 18)

les Gascons vont toujours au-delà de la vérité et

les Normands restent toujours en deçà

les Gascons aiment exagérer

(E) the natives of Gascogne have a disposi-

tion to exaggerate

(D) die Bewohner der Gascogne sind zur

Übertreibung geneigt

lessive de Gascon (17)

retourner son linge ou la nappe au lieu d'en changer

(E) turning one's underwear or the

tablecloth instead of changing it

(D) die Unterwäsche oder das Tischtuch

umdrehen anstatt zu wechseln

promesse de Gascon (19e)

promesse en l'air

(E) hollow promise

(D) leere Versprechung

s'en tirer en Gascon (17)

se tirer d'affaire par ruse

(E) to wangle one's way out of it

(D) sich durch List oder einen Kniff

herauswinden

tour de Gascon (17e)

larcin

(E) touch (NC, 19e-20 )

(D) Besitzwechsel (NC, 20")

gasconnade

1. fanfaronnade 2. cf. histoire de GASCON

(E) 1. piece of brag (NC, 19e) 2. cf. histoire de GASCON

(D) 1. Grosstuerei (NC, 18) 2. cf. histoire de GASCON 


\section{GAULE}

Gaulois

individu d'une gai eté libre et rude

(E) coarsely merry fellow

(D) derber Spassvogel

esprit gaulois

humour grivois

(E) broad Gallic humour

(D) derber Humor

histoire gauloise

plaisanterie corsée

(E) broad joke

(D) derber Witz

GORGONZOLA

avoir les dents plombées au Gorgonzola (NC, 20)

avoir une mauvaise haleine

(E) to be whiffy (NC, 1899)

(D) zehn $M$ eter gegen den Wind riechen

(NC, 20)

\section{GRAVELOTTE}

ça tombe comme à Gravelotte (1870)

1. cela arrive de partout 2 . il pleut à verse

(E) 1. it's coming from all sides 2 . it's raining cats and dogs

(D) 1. es kommt von allen Seiten 2. es giesst in Strömen

\section{GRÈCE}

la Grèce (NC, 1875)

le monde des tricheurs (aux cartes)

(E) the card-sharpers' world

(D) die Falschspielerwelt grec

1. escroc, tricheur (1752) 2. client avare de pourboire (1878)

(E) 1. Greek (1528) 2. one who tips miserly

(D) 1. Falschspieler 2. Kunde der kärgliches

Trinkgeld gibt

aux calendes grecques (16e)

sine die, jamais

(E) at/on the Greek calends (16)

(D) auf ewige Zeiten, niemals

c'est du grec pour moi ! être grec

(cf. c'est de l'ALLEM AND !)

1. être habile, rusé (1842) 2. être savant (17e)

3. être ivre (17e)

(E) 1 . to be a cute one $\left(\mathrm{NC}, 18^{\mathrm{e}}\right) 2$. to be knowledgeable 3 . to be half-seas over (NC, $\left.17^{\mathrm{e}}-20^{\mathrm{e}}\right)$

(D) 1. gewichst sein (NC, 19e) 2.

kenntnisreich sein 3. blau sein (NC, 1850)

faire le grec (NC, 19e)

trafiquer les cartes (à jouer)

(E) to work the broads

(D) die Spielkarten fälschen ne pas être grand grec $\left(17^{\mathrm{e}}\right)$

1. cf. ALLOBROGE 12 2. être peu industrieux

(E) 1. cf. ALLOBROGE 12 . not to be very skilful

(D) 1. cf. ALLOBROGE 1 2. nicht sehr geschickt sein

passez, c'est du grec! (1842)

ne vous en mêlez pas, vous n'y entendez rien

(E) don't interfere, you don't know a thing about it

(D) lassen Sie es sein, Sie verstehen gar nichts davon

va te faire voir chez les Grecs! (NC, 20")

va-t'en au diable!

(E) go to Bath (NC, 1830)/to Bungay (NC,

19)/to Hell or Connaught (NC, 1654)/to

Jericho (NC, 1635)!

(D) geh zum Teufel !

vol à la grecque (1894)

vol au change

(E) confidence-trick

(D) Wechselbetrug

\section{GRENOBLE}

faire la conduite de Grenoble à quelqu'un (1787) éconduire, renvoyer (rudement)

(E) to help s.o. along with the toe of one's boot

(D) jn energisch heimleuchten

\section{GRUYĖRE}

morceau de Gruyère (NC, 1832)

visage marqué par la vérole

(E) pitted face (by pox)

(D) blatternarbiges Gesicht

\section{GUINÉE}

nymphe de Guinée (17e)

femme noire et laide

(E) plain black woman

(D) hässliche schwarze Frau

\section{HAMBOURG}

hambourgeois (NC, 1931)

policier en civil

(E) plain-clothes policeman

(D) Polizist in Zivil

\section{HOLLANDE}

je n'ai que faire d'aller en $\mathrm{H}$ ollande, ma fortune est faite (17)

réponse à de belles promesses auxquelles on ne croit pas

(E) a reply to fine promises one does not

believe in

(D) Antwort auf schöne Versprechungen

woran man nich glaubt

\section{HONGRIE}

la petite $\mathrm{H}$ ongrie (17)

les castrats; les impuissants 
(E) castrated or impotent men

(D) Kastraten, zeugungsunfähige $M$ änner prince de Hongrie (17e)

homme châtré, impuissant

(E) castrated, impotent man

\section{INDE}

(D) Kastrat, zeugungsunfähiger $M$ ann

aller garder les poulets d'Inde (17)

épouser un nobliau campagnard

(E) to marry a country squire

(D) einen Landedelmann heiraten indien (NC, 1950)

1. malfaiteur 2. individu quelconque

(E) 1. criminal 2. any fellow

(D) 1. Verbrecher 2. jeder Beliebige

indiennes (NC, 1872)

vêtements

(E) togs (NC, 19e)

(D) Klamotten (NC, 1900)

à la file/en file indienne (19e)

à la queue leu leu

(E) in Indian file

(D) in Gänsemarsch

\section{IROQUOIS}

Iroquois

1. cf. ALLOBROGE 1 (1867)

2. cf. ALLOBROGE 2

3. individu coiffé à la punk (NC, 1986)

(E) 1. cf. ALLOBROGE 1

2. cf. ALLOBROGE 2 3. punk haircut

(D) 1. cf. ALLOBROGE 1

2. cf. ALLOBRO GE 2 3. Punkhaarschnitt C'est de l'iroquois pour moi !

(cf. c'est de l'ALLEM AND !)

\section{ISRAËL}

c'est un bon israélite (1842)

c'est un homme candide, un peu simple

(E) he's an artless, rather simple-minded fellow

(D) er ist ein redlicher, etwas alberner Mensch

\section{JAPON}

japonais (NC, 1926)

argent

(E) beans (NC, 1850)

(D) $\operatorname{Moos}\left(\mathrm{NC}, 18^{\circ}\right)$

les japonais ont tourné le coin (NC, 1926) je suis sans le sou

(E) I'm broke (NC, 1820)

(D) ich bin blank (NC, 18)

montrer ses estampes japonaises (20)

faire une invitation érotique

(E) an invitation to erotic pleasures

(D) eine erotische Einladung machen
JAVA

javanais

1. sorte d'argot (1857) 2. cf. AUVERGNAT 2

(E) 1. a sort of French slang 2. cf. AUVER-

GNAT 2

(D) 1. eine Art französische Gaunersprache

2. cf. AUVERGNAT 2

\section{JUDÉE}

la petite Judée (NC, 1847), la Judée (NC, 1866)

la préfecture de police

(E) (Paris) police headquarters

(D) (Pariser) Polizeipräsidium

juif (17e)

1. cf. ARABE 12 2. avare 3. hypocrite

(E) 1. cf. ARABE 12 . tightwad (NC, 1934)

3. shifty customer (NC, 19e)

(D) 1. ARABE 12. Pfennigfuchser (NC, 18)

3. Duckmäuser (NC, 16)

écrire à un juif (NC, 1867)

déféquer

(E) to bury a Quaker (NC, 1840)

(D) Kaktus pflanzen (NC, 20)

être entre les mains des juifs (17)

être en danger

(E) to be in danger

(D) in Gefahr sein

faire quelque chose en juif (NC, 1844)

faire qqch en se cachant, sans partager

(E) to do sthg on the sly, without sharing

(D) etwas heimlich tun, ohne zu teilen

le petit juif

os du coude sur lequel passe le nerf cubital

(E) funny bone

(D) Judenknochen

riche comme un juif (17)

très riche

(E) wallowing in money (17)

(D) mordsreich (19e)

\section{LAGNY}

être de Lagny, n'avoir pas de hâte (1640)

être très lent, indolent

(E) to be none of the $\mathrm{H}$ astings sort (16-19e)

(D) drämeln (NC, 18)

\section{LAMBALLE}

camus de Lamballe, un pied et demi de nez (17e) individu au long nez

(E) long-nosed fellow

(D) langnasiger Kerl

\section{LANDERNEAU}

landerneau (1867)

milieu étroit et fermé

(E) small, exclusive circle

(D) kleiner, geschlossener Kreis 
faire du bruit dans Landerneau (1867)

bouleverser, provoquer de l'émoi

(E) to set up the apple-cart, to make the yokels sit up

(D) Aufregung stiften, viel zu reden geben

\section{LA ROCHELLE}

il vient de La Rochelle, il est chargé de maigre

(17)

il n'a que la peau sur les os

(E) he is as thin as a lath

(D) er ist nur noch Haut und Knochen (18)

\section{LE MANS}

un Manceau vaut un Normand et demi en chicane, les habitants du Mans sont encore plus terribles que les Normands

(E) the natives of Le M ans outwrangle even those of Normandy

(D) die Bewohner von Le M ans sind noch streitsüchtiger als die von Normandien

\section{LESBOS}

lesbienne (1867)

homosexuelle

(E) lesbian (1900)

(D) Lesbierin

\section{LIMOUSIN}

les Auvergnats et les Limousins font leurs

affaires, puis celles de leurs voisins

(cf. AUVERGNE)

manger du pain comme un Limousin

être grand mangeur de pain

(E) to be a big bread eater

(D) ein tüchtiger Brotesser sein

zeste de Limousin

morceau de pain trempé dans du vin

(E) a piece of bread soaked in wine

(D) ein in Wein eingetunktes Stück Brot

\section{LOMBARDIE}

lombard (17e)

1. cf. ARABE 1. 2. trompeur 3. lâche

(E) 1. cf. ARABE 12 . cheater 3. coward

(D) 1. cf. ARABE 12 . Betrüger 3. Feigling

fuir comme un Lombard (17e)

faire preuve de lâcheté

(E) to run away like a coward

(D) feig davonlaufen

patience de Lombard (17e)

patience obligée

(E) forced patience

(D) gezwungene Geduld

\section{MACÉDOINE}

macédoine (de fruits, de légumes) (1850)

assortiment de fruits ou de légumes coupés

(E) fruit salad, macedoine of vegetables

(D) Gemisch von allerlei Früchten oder

Gemüsen

\section{MARATHON}

marathon (1896)

course à pied de 42, $195 \mathrm{~km}$

(E) marathon (race) (1896)

(D) Marathonlauf

marathon oratoire $\left(20^{\mathrm{e}}\right)$

discours interminable

(E) marathon speech $\left(20^{\circ}\right)$

(D) M arathonrede (20)

séance marathon $\left(20^{\circ}\right)$

réunion très longue

(E) marathon meeting $\left(20^{\mathrm{e}}\right)$

MAROC

(D) Marathonsitzung (20)

maroquin (1901)

portefeuille ministériel

(E) minister's portfolio

(D) M inisterposten

péter le maroquin à quelqu'un (17e)

battre avec violence

(E) to make hamburger of s.o. (US, NC)

(D) jn. zu Mus hauen (NC, 1870)

\section{MAROLLES}

pucelle de Marolles (16-17e)

(cf. pucelle de Belleville)

\section{MARSEILLE}

être de $M$ arseille

raconter des histoires exagérées, invraisem-

blables

(E) to tell Texan tales (US)

(D) Geschichten aus dem Wienerwald erzählen

marseillais (NC, 136)

revolver

(E) barker (NC, 1815)

(D) Kanone (NC, 20 )

marseillaise

1. revolver de poche $(N C, 1901) 2$. jeu de hasard (1895)

(E) 1. pocket revolver 2. game of chance

(D) 1. Taschenrevolver 2. Glückspiel

histoire marseillaise

(cf. histoire de GASCON)

raconter des histoires marseillaises

(cf. être de MARSEILLE)

\section{MAURITANIE}

traiter quelqu'un de Turc à Maure (17e)

traiter qqn durement

(E) to treat s.o. harshly

(D) jn schonungslos behandeln

\section{MOHICANS}

le dernier des $M$ ohicans

I'ultime exemplaire (bouteille, argent, etc.)

(E) the very last piece (bottle, banknote, etc.)

(D) der letzte M ohikaner (1900) 
se faire scalper le M ohican (NC, 1946)

subir une fellation

(E) to get a blow job (NC, 19e)

(D) sich blasen lassen (NC, 19e)

\section{MONACO}

du monaco, des monacos (NC, 1842-1931)

(cf. JAPONAIS)

\section{MONGOLIE}

mongol(ien) (1866)

individu atteint de trisomie 21

(E) M ongolian (person)

(D) Mongoloider

mongolisme (1866)

trisomie 21, syndrome de Down

(E) Kalmuk/M ongolian idiocy, mongolism

(D) M ongolismus

\section{NANTERRE}

arriver comme les pompiers de $N$ anterre

arriver trop tard

(E) to come a day after the fair

(D) einen Posttag zu spät kommen

\section{NAPLES}

aller à Naples sans passer par les monts (17)

(cf. aller en BAVIÈRE)

mal de Naples (17)

(cf. mal ESPAGNOL)

voir Naples et (puis) mourir (19e)

expression d'un ardent souhait

(E) voicing of an eager wish

(D) Ausdruck eines heissen Wunsches

mal napolitain (17)

(cf. mal ESPAGNOL)

\section{NICE}

être niçois (NC, 1953)

ne pas faire de gains au poker

(E) to collect no winnings at poker

(D) beim Pokerspiel keinen Gewinn machen

\section{NIORT}

aller à Niort, prendre le chemin de Niort (17e)

nier
(E) to deny
(D) leugnen

\section{NORMANDIE}

il s'est donné à plus de diables qu'il n'y a de pommes en Normandie (17)

il a fait un grand serment

(E) he has taken a solemn oath

(D) er hat sich eidlich verpflichtet

à Normand, Normand et demi

à trompeur, trompeur et demi

(E) set a thief to catch a thief

(D) auf einen Schelm anderthalben

$c^{\prime}$ est un fin Normand (17')

c'est un homme rusé
(E) he's as cunning as a Yorkshireman (19e-20e)

(D) er ist ein schlauer Fuchs

être adroit comme un prêtre normand

être très maladroit

(E) to be all thumbs (NC, 19)

(D) zwei linke Hände haben (NC, 19e)

être normand (17)

1. cf. être GREC 2. se dédire

(E) 1. cf. être GREC 2. to go back on one's word

(D) 1. cf. être GREC 2. Sein Wort zurücknehmen

faire une réponse de Normand, répondre en

Normand (17e)

ne dire ni oui ni non, ne pas s'engager

(E) to give an evasive answer

(D) eine ausweichende Antwort geben

il ressemble les Normands, il a son dit et son dédit

il revient facilement sur sa parole

(E) he readily backs out

(D) er kommt leicht auf sein Wort zurück les Gascons vont toujours au-delà de la vérité, les $N$ ormands restent toujours en deçà

(cf. GASCOGNE)

qui fit Normand, fit truand (1842)

la pauvreté pousse à la mendicité

(E) poverty leads to begging

(D) Armut führt zum Bettelstand

réconciliation normande

réconciliation simulée

(E) feigned reconciliation

(D) angebliche Versöhnung

un $M$ anceau vaut un $N$ ormand et demi

(cf. LE MANS)

un Normand a son dit et son dédit

(cf. il ressemble les N ORM ANDS)

\section{ORLÉANS}

c'est de la glose d'Orléans (17)

l'explication est plus obscure que le texte

(E) the explanation is more difficult to

understand than the text

(D) die Erklärung ist schwieriger zu

verstehen als der Text

être de l'abbaye des luniers d'O rléans (17e)

être lunatique, inconstant

(E) to be whimsical, fickle

(D) wankelmütig, grillenhaft sein

\section{OSTROGOTHS}

ostrogoth (17e)

1. cf. ALLOBROGE 12 2. cf. ALLOBROGE 2

(E) 1. cf. ALLOBRO GE 1

2. cf. ALLOBROGE 2

(D) 1. cf. ALLOBROGE 12.

cf. ALLOBROGE 2 


\section{PAMPELUNE}

envoyer à Pampelune (17)

(cf. envoyer à CANCALE)

\section{PARIS}

Paris appartient à ceux qui se lèvent tôt il ne faut point paresser si l'on veut réussir

(E) it's the early bird that catches the worm

(D) Morgenstund hat Gold im Mund

Paris ne s'est pas fait en un jour (17) rien ne peut se faire sans le temps voulu

(E) Rome was not built in a day (16)

(D) Rom ist nicht an einem Tage erbaut worden

Paris vaut bien une messe (16e)

sacrifier à son intérêt ce qui est son devoir

(E) to sacrifice one's duty to one's interest

(D) seine Pflicht seinem Interesse opfern

avec des si, on mettrait Paris en bouteille (18e) avec des hypothèses, tout devient possible

(E) with assumptions, everything becomes possible

(D) mit Voraussetzungen wird alles möglich avoir un œil à Paris et l'autre à Pontoise

(cf. regarder en Gâtinais pour voir si la

Champagne brûle)

chou pour chou, Aubervilliers vaut bien Paris

(cf. AU BERVILLIERS)

faire comme on fait à Paris (17)

laisser pleuvoir (sans s'en soucier)

(E) not to care about rain

(D) sich um den Regen nicht kümmern

il y a des lieux où il faut appeler Paris Paris,

et d'autres où il la faut appeler capitale du

royaume (1670)

il faut s'adapter à son entourage

(E) one must adapt to one's environment

(D) man soll sich seiner Umgebung anpassen

Monsieur de Paris (19e)

le bourreau

(E) the executioner

(D) der Henker

prendre Paris pour Corbeil

(cf. CORBEIL)

si Paris avait une Cannebière, ce serait un petit

Marseille

dicton attribué par moquerie aux $M$ arseillais

(E) a saying ascribed derisively to the people of $M$ arseilles

(D) ein scherzhaft den Leuten von Marseille zugeschriebenes Sprichwort

Paris est le paradis des femmes, le purgatoire des hommes, l'enfer des chevaux (19e)

les femmes s'amusent, les hommes travaillent, les chevaux crèvent de fatigue
(E) women have fun, men work, horses die of exhaustion

(D) die Frauen vergnügen sich, die M änner arbeiten, die Pferde sterben an Ermüdung

si Paris était de beurre, il fondrait au soleil (17e) réponse à une supposition absurde

(E) a reply to a stupid assumption

(D) Antwort auf eine dumme Vermutung

\section{PAYS-BAS}

Pays-Bas

1. sexe de la femme (NC, 1863) 2. cf. BAS-

RHIN (NC, 1867)

(E) 1. Berkeley (Hunt) (NC, 20) 2. cf. BAS-

RHIN

(D) 1. Fotze (NC, 18) 2. cf. BAS-RHIN

\section{PÉKIN}

pékin

1. bourgeois (1776) 2. civil (NC, 1799)

(E) 1. Philistine (1827) 2. civvy (NC, 1895)

(D) 1. Philister 2. Philister

en pékin

en vêtements civils

(E) in mufti (NC, 1850)

(D) in Zivilkleidung

\section{PÉROU}

pérou (17)

objet de grande valeur, grosse fortune

(E) valuable object, big fortune

(D) wertvolle Sache, grosses Vermögen

ce n'est pas le Pérou! (1867)

c'est de peu de profit

(E) it's no great catch! (NC, 20)

(D) damit wirst du nicht Amerika machen!

gagner le Pérou (17)

faire fortune

(E) to make a fortune

(D) eine grosses Vermögen erwerben

\section{PHILISTINS}

philistin (1832)

bourgeois à l'esprit fermé aux arts, aux

lettres, aux nouveautés

(E) philistine (1827)

(D) Philister (19e)

\section{PICARDIE}

regarder en Picardie pour voir si la Champagne brûle

(cf. CHAM PAGNE)

il a ressemblé le Picard (17)

il l'a échappé belle

(E) he had a narrow escape

(D) er ist mit knapper Not davongekommen

\section{POISSY}

olives de Poissy (NC, 17)

testicules 
(E) cobblers (NC, 20 $)$

(D) Eier (NC, 1900)

\section{POITIERS}

roi de Poitiers (17e)

dignité qui ne dure pas

(E) short-lived high position

(D) kurzlebige hohe Stellung

\section{POLOGNE}

boire comme un Polonais (19e)

boire sec

(E) to booze (NC, 16-20)

(D) saufen wie ein Loch (NC, 18')

gris/saoul comme un Polonais (19e)

complètement ivre (cf. être GREC)

(E) as drunk as a Dutchman, as merry as a Greek (US)

(D) blau wie ein Veilchen (NC, 1914-18)

\section{PONTOISE}

avoir un œil à Paris et l'autre à Pontoise

(cf. PARIS)

mettre Villejuif dans Pontoise (17e)

(cf. jouer au billard ANGLAIS)

revenir de Pontoise (1842)

avoir l'air ahuri, tomber de la lune

(E) to have been wool-gathering (16e)

(D) verträumt sein

\section{PORTUGAL}

portugaises (NC, 1951)

oreilles

(E) lugs (NC, 16e-20e)

(D) Löffel (NC, 19e)

avoir les portugaises ensablées (NC, 1951)

1. être sourd 2. ne pas comprendre

(E) 1. to be deaf 2. to fail to understand

(D) 1. taub sein 2. einen Knoten im Strom haben (NC, 20)

embouteiller les portugaises à quelqu'un (NC, 1957)

casser les oreilles à qqn

(E) to split s.o.'s ears

(D) jm. die Ohren vollreden

\section{PROVENCE}

borgne de Provence (1842)

aveugle

(E) blind man

(D) blinder Mann

\section{PRUSSE}

aller voir le roi de Prusse

aller aux toilettes

(E) to give the Chinaman a music lesson

(US, NC), to go to Egypt (US, NC, 20)

(D) zu Tante Meier gehen (NC, 1850)

travailler pour le roi de Prusse (1845)

travailler gratuitement

(E) to work for nothing
(D) umsonst arbeiten

prussien (NC, 19e)

(cf. BAS-RHIN)

\section{RENNES}

faire l'épaule rennoise à qqn $\left(20^{\circ}\right)$

bouder qqn

(E) to give s.o. the cold shoulder

(D) jm. die kalte Schulter zeigen

\section{ROME}

Rome ne s'est pas faite en un jour (16e)

(cf. PARIS)

à Rome, il faut vivre comme à Rome (4)

il faut se conformer aux usages du pays

(E) at Rome you must do as the Romans do

(D) in Rom, tu' wie Rom tut

aller/passer à Rome

se faire gronder ou corriger

(E) to get a scolding or beating

(D) eine Rüge oder eine Züchtigung

bekommen

fou à Rome va, fou en revient

on ne se corrige pas en voyageant

(E) send a fool to the market and a fool he'll return

(D) schick den Esel nach Oxford, nimmer wird ein Pferd er dort

il vaut mieux être le premier dans son village que le second dans Rome

la gloire locale est préférable

(E) better to reign in $\mathrm{Hell}$ than to serve in Heaven

(D) lieber der erste hier als der zweite in Rom

jamais cheval ni méchant homme n'amenda pour aller à Rome

(cf. fou à ROME va...)

qui bête va à Rome, tel en retourne

(cf. fou à ROME va...)

qui langue a, à Rome va

quand on sait s'expliquer, on peut aller partout

(E) a ready tongue will bring you anywhere

(D) wer ein gutes Mundwerk hat kommt überall durch

si jamais cela arrive, je l'irai dire à Rome! se dit à propos d'une chose impossible où improbable

(E) if ever that happens, I'll eat my hat!

(D) ich will Meier heissen wenn das passieren soll!

s'il n'est pas content, qu'il aille le dire à Rome! peu m'importe son déplaisir

(E) if he doesn't like it, he can lump it!

(D) er wird in den sauren Apfel beissen müssen! 
tout chemin mène/tous les chemins mènent à Rome

il y a maints moyens d'arriver au même but

(E) all roads lead to Rome

(D) alle Wege führen nach Rom Romain

1. homme austère, courageux (17e) 2. joueur qui a tout perdu (NC, 1833) 3. individu payé pour applaudir (NC, 1828)

(E) 1. a stern, courageous man 2. one who has lost everything at games 3 . one paid to applaud

(D) 1. edler, mutiger $M$ ensch 2. einer der beim Spiel alles verloren hat 3 . bezahlter Klatscher

le dernier des Romains (1835) homme d'une grande autorité morale

(E) a lofty moral authority

(D) moralisch einflussreiche angesehene Person

travail de Romain (1907)

travail difficile, pénible

(E) Trojan work

(D) beschwerliche Arbeit

romaine

claque, coup

(E) clout (NC, 18)

(D) $\mathrm{Hau}\left(\mathrm{NC}, 16^{\mathrm{e}}\right)$

bon comme la romaine (1915)

d'une bonté à toute épreuve

(E) (too) good-natured

ROUEN

(D) (zu) gutmütig

aller à Rouen (1872)

1. aller à sa perte 2 . se faire siffler

(E) 1. to head for disaster 2. to get the bird

(D) 1 . in sein Verderben rennen 2. ausgepfiffen werden

faire un Rouen (19)

rater une vente

(E) to miss a sale

(D) ein Geschäft verpassen

\section{RUSSIE}

russe (NC, 1910)

recrue

(E) rookie (NC, 1893)

(D) Hammel (NC, 18)

chaussettes russes (20)

linges enveloppant les pieds

(E) Russian socks (NC, 1935)

(D) Fusslappen

roulette russe (20e)

choix hasardeux

(E) Russian roulette

(D) gewagte Wahl

\section{SAINT-CLOUD}

marguillier de Saint-Cloud (1640)

nigaud

(E) wise man of Gotham (1694)

(D) Einfaltspinsel (NC, 18)

\section{SAINT-MALO}

il a été à Saint-M alo (1842)

il n'a pas de mollets

(E) he has legs like drumsticks (NC, 18)

(D) Streichholzbeine haben (NC, 19)

\section{SAINT-MICHEL}

vendre des coquilles à ceux qui viennent

de Saint-M ichel (16 $)$

faire quelque chose d'inutile

(E) to carry coals to Newcastle

(D) Eulen nach Athen tragen

\section{SAMARIE}

jouer au bon Samaritain (19e)

se dévouer

(E) to be a good Samaritan

(D) den barmherzigen Samariter spielen

frère/officier de la Samaritaine (17e)

proxénète

(E) ponce (NC, 1872)

(D) Schlurf (NC, 1922)

\section{SIAM}

être comme des frères siamois (1872)

être inséparables

(E) to be like Siamese twins (1833)

(D) unzertrennliche Freunde sein

\section{SIOUX}

sioux (NC, 1952)

militaire américain

(E) U.S. soldier

(D) amerikanischer Soldat

avancer avec des ruses de Sioux

de manière furtive

(E) to proceed stealthily

(D) verstohlenerweise vorgehen

ruses de Sioux

stratagèmes

(E) stratagems, scheming

(D) Liste, Machenschaften

\section{SODOME}

c'est Sodome et Gomorrhe

c'est un endroit immoral, corrompu

(E) it's a regular Sodom

(D) es ist ein wahres Sodom und Gomorra sodomie (13e)

(cf. botte FLORENTINE)

\section{SOISSONS}

soissonnais (NC, 1928), soissonnais rose (NC, 1957), soissons (NC, 1901)

clitoris

(E) the little man in the boat (NC, 19e-20e)

(D) Kitzler 


\section{SOLOGNE}

niais de Sologne (17)

qui simule la niaiserie pour duper

(E) one who pretends to be silly in order to dupe others

(D) jemand der sich dumm stellt um andere zu prellen

\section{SOVIÉTIQUES}

whisky soviétique (NC, 1984)

verre de vin rouge

(E) glass of red wine

(D) Glas Rotwein

\section{SUĖDE}

aller en Suède (17)

faire soigner sa syphilis

(E) to have one's pox treated

(D) Schwitzkur gegen Syphilis gebrauchen

\section{SUISSE}

autant vouloir parler à un Suisse! (17e)

c'est peine perdue!

(E) you might as well talk to a brick wall!

(D) das ist tauben Ohren predigen!

boire comme un Suisse (17')

(cf. boire comme un POLONAIS)

boire en Suisse (1867)

boire seul

(E) to go to Ballarat (NC, 1890-1930)

(D) einsam trinken

c'est un amiral suisse

il n'a jamais vu la mer

(E) he's a Swiss admiral $(1870,0 b)$

(D) er ist von der reitenden Gebirgsmarine (NC)

faire Suisse

1. boire ou manger seul (NC, 1841) 2. vivre en égoïste (NC, 1863)

(E) 1. to drink or eat by oneself 2 . to act selfishly

(D) 1. einsam essen oder trinken 2. selbstsüchtig verfahren

n'entendre pas plus raison qu'un Suisse ne tenir compte d'aucune observation

(E) to refuse to see reason

(D) keine Belehrung annehmen

parler à un Suisse (17e)

parler à un mur

(E) to talk to a brick wall

(D) tauben Ohren predigen

point d'argent, point de Suisse! (17)

rien pour rien

(E) no pay, no piper

(D) umsonst ist der Tod!

rêver à la Suisse

ne penser à rien
(E) to think of nothing

(D) im Leerlauf denken (NC, 20)

ventre à la Suisse (17)

gros ventre

(E) German/M ilwaukee goiter (US, NC)

(D) Kartoffelbauch (NC, 1900)

\section{TOLÈDE}

de Tolède (1872)

de première qualité

(E) gorgonzola $\left(\mathrm{NC}, 20^{\mathrm{e}}\right)$

(D) prima (19e)

\section{TUNISIE}

sourire tunisien (NC, 1984)

balafre

(E) scar (in the face)

(D) Schmarre

\section{TURQUIE}

bigar ré comme un tapis de Turquie (17e)

vêtu de diverses couleurs

(E) many-coloured like a Turkish carpet

(D) buntgekleidet

jouer des orgues de Turquie $\left(17^{\mathrm{e}}, \mathrm{ob}\right)$

manger

(E) to grub (NC, 18)

(D) einfahren (NC, 1900)

$\operatorname{Turc}(17 \mathrm{e}, \mathrm{ob})$

1. homme cruel, impitoyable

2. cf. ALLOBROGE 2

(E) 1. harsh, cruel man 2. cf. ALLOBROGE 2

(D) 1. grausamer, schonungsloser $M$ ensch

2. cf. ALLOBROGE 2

à la turque (19e)

durement, rudement

(E) harshly, roughly

(D) schonungslos

face du Grand Turc (NC)

(cf. BAS-RHIN)

fort comme un Turc (1690)

d'une grande force physique

(E) as strong as an ox

(D) stark wie ein Pferd

jeunes Turcs (1908)

jeunes militants, partisans d'une évolution dans un parti politique

(E) young Turks (19e)

(D) Jungtürken

les amis ne sont pas des Turcs

l'amitié est indulgente

(E) friendship is condoning

(D) Freundschaft ist nachsichtig

s'en soucier comme du Grand Turc (19e)

ne faire aucun cas de qqch

(E) not to care a rap (1834)

(D) sich den Teufel um etwas scheren (18) 
tête de Turc (1867)

souffre douleur, bouc émissaire

(E) scapegoat (16e)

(D) Sündenbock

traiter de Turc à M aure/M ore (17) (cf. MAURITANIE)

travailler pour le Grand Turc (19e)

(cf. travailler pour le roi de PRUSSE)

\section{VANDALES}

vandale (1732)

destructeur (de belles choses)

(E) vandal (1752)

(D) Vandale

vandalisme (1739)

esprit de destruction, action de détruire

(E) vandalism (1787)

(D) Vandalismus

\section{VANVES}

il est sur le four de Vanves $\left(17^{e}, o b\right)$

il s'est égaré

(E) he lost his way

(D) er hat sich verirrt

\section{VENISE}

cataplasme de Venise (1867)

(cf. ROM AINE)

secours des Vénitiens $\left(17^{e}\right.$, ob.)

secours tardif

(E) belated help

(D) späte Hilfe

VILLEJUIF

mettre Villejuif dans Pontoise (17e)

(cf. PONTOISE)

WATERLOO

Waterloo (NC, 1879, ob.)

(cf. BAS-RHIN)

rencontrer son Waterloo (19)

subir un échec decisif

(E) to meet one's Waterloo

(D) eine entscheidende Niederlage erleiden

\section{ZANZIBAR}

brouette de Zanzibar (NC, 18)

position de coït

(E) a love-making posture

(D) eine Stellung beim Geschlechtsverkehr 


\section{LEXIQUE D'EXPRESSIONS ANGLAISES}

\section{ABERDEEN}

Aberdeen cutlet (NC, 1870, ob.)

dried haddock

(F) aiglefin séché

(D) getrockneter Schellfisch

\section{ABERGAVENNY}

Abergavenny (RS, 1880)

penny coin

(F) pièce d'un penny

(D) Pennystück

\section{ABYSSINIA}

Abyssinia! (NC, 20)

I'll be seeing you !

(F) à bientôt!

(D) auf Wiedersehen!

Abyssinian medal (NC, 1896-1914)

showing fly button

(F) bouton de braguette apparent

(D) sichtbarer Hosenschlitzknopf

\section{ACAPULCO}

Acapulco (gold) (US, NC, 20)

marihuana

(F) marijuana

(D) Marihuana

\section{AFRICA}

African dominoes (US, NC, 1920), African golf

(US, NC, 20)

dice
$(F)$ jeu de dés

(D) Würfelspiel

African harp (NC, 1930)

banjo

(F) banjo

(D) Banjo

African woodbine (NC, 20)

marihuana cigarette

(F) cigarette de marijuana

(D) Marihuanazigarette

\section{ALABAMA}

Alabama wool (US, NC)

pure cotton

(F) pur coton

(D) reine Baumwolle

\section{ALSATIA}

Alsatia (NC, 1680-1800)

a low quarter of London where bankrupts,

criminals, etc. found shelter

$(F)$ bas quartier de Londres où les banqueroutiers, criminels, etc. trouvaient refuge

(D) Londener Viertel wo Bankbrüchiger,

Verbrecher, usw. sich in Sicherheit brachten

\section{AMERICA}

America's Boating Capital (US)

the City of Seattle

(F) la ville de Seattle

(D) die Stadt Seattle

America's Dairyland (US)

the State of Wisconsin

(F) l'État du Wisconsin

(D) der Staat Wisconsin

America's H eartland (US)

the State of Iowa

(F) l'État d'lowa

(D) der Staat lowa

Athens of America (US)

the City of Boston

(F) la ville de Boston

(D) die Stadt Boston

Beer Capital of America (US)

the City of Milwaukee

(F) la ville de Milwaukee

(D) die Stadt M ilwaukee

Crossroads of America (US)

the State of Indiana

(F) l'État d'Indiana

(D) der Staat Indiana

Switzerland of America (US)

the State of M aine

(F) l'État du Maine

(D) der Staat Maine

American letter (NC, 19)

condom

(F) préservatif ; capote anglaise (NC, 1878)

(D) Präservativ; Pariser (NC, 1956)

\section{ARABIA}

Arab

1. violent, dissolute man (NC, 1850)

2. street-hawker (US, NC, 1935)

(F) 1. individu violent, dissolu 2. marchand des quatre saisons

(D) 1. gewaltsamer, liederlicher Kerl 2.

umhergehender O bst- und Gemüsehändler

street Arab (NC, 1855)

street urchin, guttersnipe

(F) jeune vagabond, gamin des rues

(D) Gassenjunge

\section{ARCADIA}

Arcadian (1667)

ideally rustic, idyllic

(F) d'une simplicité pastorale, idyllique; arcadien

(D) einfach ländlich, friedlich; arkadisch 
Arcadian days

days of innocence and happiness

(F) jours d'innocence et de bonheur

(D) friedliche, glückliche Tage

Arcadian nightingale (NC, 19, ob.)

1. donkey 2. ignoramus

(F) 1. âne; rossignol/roussin d'Arcadie (NC, 1842 , ob.) 2. ignorant; iroquois (NC, 1867, ob.)

(D) 1. Esel 2. Dummkopf; Vollblutindianer (NC, 1920)

ARGYLL

Duchess of Argyll (RS, 20)

file

(F) lime

(D) Feile

\section{ARIZONA}

Arizona tenor (US, NC)

tuberculous cough

(F) toux tuberculeuse

(D) schwindsüchtiges Husten; letzte Grüsse aus Davos (NC)

\section{ARKANSAS}

Arkansas chicken (US, NC) salted pork

(F) porc salé

(D) eingesalzenes Schweinefleisch

Arkansas lizard (US, NC, ob.)

louse

(F) pou

(D) Laus

Arkansas toothpick (US, NC, 1840)

cutlass, bowie-knife

(F) coutelas, couteau-poignard

(D) H irschfänger

ATHENS

Athens of America

(cf. America)

ATTICA

Attic faith (17)

never-failing fidelity

(F) fidélité à toute épreuve; foi attique

(D) ewige Treue

Attic salt/wit (17)

caustic wit

(F) esprit caustique; sel attique

(D) beissender Witz; attisches Salz

\section{AZTECS}

Aztec idiocy

microcephalic idiocy

(F) idiotie microcéphalique

(D) Idiotie der Mikrocephalen

Aztec two-step (US, NC)

diarrhea
(F) diarrhée

(D) Durchfall

\section{BABYLONIA}

Babylonian (1564)

1. gigantic 2. sumptuous

(F) 1. gigantesque 2. somptueux, babylonien

(D) 1. riesenhaft 2. prunkvoll

\section{BALLARAT}

to go to Ballarat (NC, 1890-1930)

to drink alone

(F) boire seul ; boire en Suisse (19)

(D) einsam trinken

BATH

go to Bath! (NC, 1830)

go to hell!

(F) va-t'en au diable! va te faire voir chez les Grecs (NC, 1958)

(D) scher dich zum Teufel !

\section{BATTERSEA}

to go to Battersea to be cut for the simples (NC, 18-20)

to be cured of one's madness

(F) être guéri de sa folie

(D) von seinem Wahnsinn geheilt sein

BEDFORDSHIRE

to go to Bedfordshire (NC, 17-20, ob.)

to go to bed

(F) aller au lit

(D) zu Bett gehen; nach Bettenhausen (NC,

1880)/Kissingen (NC, 1900) gehen

\section{BENGAL}

Bengal blanket (NC, 19-20, ob.)

1. the sun 2. blue sky

(F) 1. soleil; bourguignon (NC, 1821), feu des Espagnols (17, ob.) 2. ciel bleu

(D) 1. die Sonne 2. blauer Himmel

\section{BERKELEY}

berkeley (NC, 20)

1. the female genitals 2 . fool

(F) 1. sexe de la femme; Pays-Bas (NC,

1863) 2. imbécile

(D) 1. die weiblichen Genitalien 2. Dummkopf

berkeleys (NC, 1875)

female breasts

(F) seins

(D) Brüste

Berkeley Hunt (RS, 20)

(cf. berkeley 1)

BERKSHIRE

Berkshire Hunt (RS, 19-20)

(cf. berkeley 1 ) 


\section{B๔OTIA}

Bœotian (1598)

stupid, dull-witted

(F) stupide, obtus; béotien

(D) dumm, blöde

\section{BOHEMIA}

Bohemia (1861)

unconventional artist's life

(F) existence non conventionnelle, vie d'artiste; bohème (1838)

(D) argloses Künstlerleben; Bohemeleben, Bohemewesen

Bohemian

1. gipsy (1696) 2. one who leads an irregular life (1848)

(F) 1. tzigane; bohémien (15) 2. qui mène une vie irrégulière; bohème (1838)

(D) 1. Zigeuner 2. einer der ein Bohemeleben führt

to lead a Bohemian life (1861)

to have an unconventional life

$(F)$ avoir une existence irrégulière, précaire; mener une vie de bohème (1838)

(D) ein sorgloses, unsicheres Leben führen ; ein Bohemeleben führen

\section{BOLIVIA}

Bolivian marching powder (US, NC, 20)

cocaine

(F) cocaine

(D) Kokain

BOMBAY

Bombay cruds (NC, 19)

(cf. Aztec two-step)

BOSTON

Boston strawberries (US, NC, 1884)

beans

(F) haricots

(D) Bohnen

BRID PORT

Bridport dagger (NC, 17-19)

hangman's noose

(F) nœud de potence; salade de Gascon/

Gascogne (17)

(D) Strang

to be stabbed with a Bridport dagger (NC, 17-19)

to be hanged

(F) être pendu

(D) an den Galgen kommen

\section{BRIGHTON}

Brighton pier

1. queer (RS, 19-20) 2. pederast (RS, 1940)

(F) 1. bizarre 2. homosexuel

(D) 1. merkwürdig 2. Homosexueller

bike ride to Brighton (NC, 20)

sexual intercourse
(F) acte charnel ; combat de Cythère (1861),

voyage à Cythère (1864)

(D) Geschlechtsverkehr

\section{BRISTOL}

Bristol Cities (RS, 20), bristols

(cf. berkeleys)

Bristol milk (NC, 1660-20)

sherry

(F) sherry

(D) Sherry

Bristol stone

1. false diamond (NC, 17-18) 2. rock crystal (20)

(F) 1. faux diamant 2. cristal de roche

(D) 1. falscher Diamant 2. Bergkristall

\section{BRITAIN}

British champagne (NC, 1810-40)

dark beer

(F) bière brune anglaise

(D) englisches dunkles Bier

as British as beef

staunch Briton

(F) Anglais bon teint

(D) hundertprozentig Englisch

unable to say British Constitution (NC, 19-20,

ob.)

completely drunk

(F) complètement ivre; saoul comme un

Polonais/comme toute la Pologne (NC, 18)

(D) völlig betrunken

\section{BRUNSWICK}

Brunswick (US, NC, 20)

1. bottom 2. anus

(F) 1. derrière; abbaye de Cluny (NC, 1864, ob.), Bas-Rhin (NC, 19), Pays-Bas (NC,

1867), waterloo (NC, 1879, ob.) 2. anus; trou d'Aix (NC, 1864, ob.), dahoméen (NC, 1912)

(D) 1. Gesäss 2. After

\section{BRUSSELS}

Brussels sprout (RS, 1910)

boy scout

(F) scout

(D) Pfadfinder

\section{BUCKLEBURY}

Bucklebury (NC, 1923)

anal coition

(F) coït anal ; botte florentine (17), sodomie

(13)

(D) Analkoitus; Sodomiterei

\section{BUENOS AIRES}

to go to/to take the road to Buenos Aires (NC, 20)

to become a prostitute

(F) se prostituer

(D) sich prostituieren 


\section{BUNGAY}

go to Bungay! (NC, 19)

(cf. go to Bath!)

he's been to Bungay fair and broken both his legs (NC, 19)

he is drunk

(F) il est ivre; il est plein comme toute la Pologne (NC, 19)

(D) er ist völlig betrunken

\section{BURFORD}

to take a Burford bait (NC, 18-20)

to get drunk

(F) s'enivrer

(D) sich betrinken

\section{BURLINGTON}

Burlington Hunt (RS, 20)

(cf. berkeley 1)

\section{BURMA}

Burma shave (US, NC, 20)

nonsense, empty talk

(F) balivernes

(D) Quatsch

\section{BURTON-ON-TRENT}

Burton(-on-Trent) (RS, 1880)

rent
(F) loyer
(D) Miete

CALIFORNIA

California bible/prayer book (US, NC, 1855) playing cards

(F) cartes à jouer

(D) Spielkarten

California collar (US, NC, 19)

(cf. Bridport dagger)

California cornflake (US, NC, 20)

(cf. Bolivian marching powder)

California kiss-off (US, NC, 20)

(notice of) dismissal

(F) (lettre de) renvoi

(D) Entlassung (sbrief)

California sunshine (US, NC, 20)

LSD drug

(F) la drogue LSD

(D) das Rauschgift LSD

California toothpick (US, NC, 1850)

(cf. Arkansas toothpick)

California widow (US, NC, 1875)

deserted wife

(F) épouse abandonnée

(D) verlassene Ehefrau

\section{CAMBRIDGE}

Cambridge fortune (NC, 17-19)

what Nature gave to a woman

(F) femme qui a son corps pour toute fortune
(D) der Körper als ganzes Habe einer Frau

Cambridge oak (NC, 18-20, ob.)

willow

(F) saule

(D) Weide

\section{CAMBRIDGESHIRE}

Cambridgeshire camel ( NC, 17-19)

a native or resident of Cambridgeshire

(F) natif ou habitant du Cambridgeshire

(D) Eingeborener oder Einwohner vom

Cambridgeshire

Cambridgeshire nightingale (NC, 19-20)

frog

(F) grenouille

(D) Frosch

\section{CANADA}

Canadian black (US, NC, 20)

(cf. Acapulco)

Canadian quail (US, NC, 20)

the narcotic methaqualone

(F) la drogue méthaqualone

(D) das Rauschgift Methaqualon

\section{CANOSSA}

to go to Canossa (19)

to eat humble pie

(F) s'incliner honteusement; aller à Canossa

(19)

(D) sich demütigen ; nach Kanossa gehen, den Kanossaweg antreten

\section{CANTERBURY}

Canterbury tale (NC, 16, ob.)

long and tedious story

(F) histoire longue et ennuyeuse

(D) langweilige Geschichte

\section{CAPE}

Cape nightingale (NC, 1880)

(cf. Cambridgeshire nightingale)

\section{CAPE HORN}

Cape Horn $(R S, 20)$

dawn

(F) aurore

(D) Morgenrot

Cape Horn rainwater (NC, 19-20)

rum

(F) rhum

(D) Rum

to double Cape Horn (NC, 18-19)

to be made a cuckold

(F) aller en Cornouailles (17e)

(D) zum Hahnrei gemacht werden

\section{CAPE OF GOOD HOPE}

Cape of Good Hope (RS, 1914)

soap

(F) savon

(D) Seife 


\section{CAPUA}

to sink in Capuan luxury

to waste time that could be used to advantage

(F) perdre du temps que l'on pourrait

employer avantageusement

(D) Zeit vergeuden, die man recht gut benutzen könnte

\section{CARTHAGE}

Carthaginian faith (17)

treachery

(F) traîtrise; foi carthaginoise

(D) Verräterei ; punische Treue

\section{CHARING CROSS}

Charing Cross (RS, 1857)

horse

(F) cheval

(D) Pferd

\section{CHATHAM}

Chatham and Dover (RS, 1909)

(to give) over

(F) abandonner, renoncer

(D) aufgeben, verzichten

\section{CHELSEA}

it's Chelsea College to a sentry-box (NC, 1819) it's a certainty

(F) c'est une (quasi) certitude

(D) das ist eine todsichere Sache

\section{CHELTENHAM}

Cheltenham bold (RS,19-20)

cold

(F) froid(ure)

(D) Kälte

\section{CHESHIRE}

it's the Cheshire! (NC, 1870-1900)

the correct thing, perfection

(F) c'est exactement ce qu'il faut, c'est parfait

(D) es ist die richtige Sache

to grin like a Cheshire cat (1770)

to smile from ear to ear

(F) sourire jusqu'aux oreilles

(D) übers ganze Gesicht grinsen

\section{CHICAGO}

Chicago atomizer/chopper (US, NC, 20)

machinegun

(F) mitraillette

(D) Maschinenpistole

Chicago green (US, NC, 20)

(cf. Acapulco)

Chicago overcoat (US, NC, 1929)

coffin

(F) cercueil ; canadienne en sapin (NC, 20)

(D) Sarg

\section{CHINA}

China (NC, 20)

friend
(F) copain

(D) Freund

China chin (US, NC, 1950)

unsuccessful boxer

(F) boxeur médiocre

(D) schlechter Boxer

China clipper (US, 1945)

washer-up

(F) plongeur (de vaisselle)

(D) (Geschirr) Abwäscher

China's cow (NC, 20)

soja

(F) soja

(D) Soja

China plate (RS, 1870)

(cf. China)

China white (US, NC, 20)

heroin

(F) héroïne

(D) Heroin

I'd like to have you on a slow boat to China

(NC, 20)

I'd like to have time to persuade you

(F) j'aimerais avoir le temps de vous persuader

(D) ich möchte Zeit haben, Sie zu überreden

it's (all) Lombard Street to a China orange (NC, 1869)

(cf. it's Chelsea College to a sentry-box)

not for all the tea in China! (NC, 1890)

certainly not! not at all!

(F) pas pour tout l'or du monde!

(D) für nichts in der Welt!

old China (NC, 20)

old friend

(F) vieil ami

(D) alter Freund

Chinaman's shout (NC, 20)

an entertainment where each pays his part

(F) réception, repas, sortie où chacun paie sa part; invitation à l'américaine

(D) gemeinsames Vergnügen wobei jeder für sich bezahlt; Amerikanische Einladung

he hasn't got a Chinaman's chance (1849)

he has no chance at all

(F) il n'a pas I'ombre d'une chance

(D) er hat nicht die geringste Chance

to give the Chinaman a music lesson (US, NC)

to go to the toilet

(F) aller aux toilettes; aller voir le roi de Prusse (19)

(D) zur Toilette gehen

to kill the Chinaman (US, NC)

to make a lot of money

(F) gagner beaucoup d'argent, faire un gros coup 
(D) viel Geld verdienen

Chinese compliment (1880)

pretended deference to the opinion of another

(F) respect feint pour l'opinion d'autrui

(D) vorgegebener Respekt für jemandes M einung

Chinese deal (US, NC)

deal that comes to nothing

(F) affaire qui n'aboutit pas

(D) Geschäft das zu Wasser wird

Chinese dominoes (NC, 1930)

load of bricks

(F) chargement de briques

(D) Ladung Ziegelsteine

Chinese gunpowder (NC, 1930)

load of cement

(F) chargement de ciment

(D) Ladung Zement

Chinese landing (US, 1945)

landing with a damaged wing

(F) atterrissage avec une aile endommagée

(D) Landung mit einem beschädigten Flügel

Chinese needlework (US, NC)

drug injections

(F) piqûres de stupéfiants

(D) Rauschgiftspritzen

Chinese puzzle (US, NC)

difficult problem

(F) problème difficile; casse tête chinois

(D) kopfzerbrechendes Problem

Chinese red (US, NC, 20)

(cf. China white)

Chinese rocks (NC, 20)

(cf. China white)

Chinese Rolls-Royce (1914-18, ob.)

Ford car

(F) voiture Ford

(D) Fordwagen

Chinese saxophone (1910)

opium-pipe

(F) pipe à opium

(D) O piumpfeife

Chinese tobacco (US, NC, 1950)

opium

(F) opium

(D) Opium

Chinese wedding-cake (NC, 20)

rice pudding

(F) gâteau de riz

(D) Reiskuchen

in the words of the Chinese poet (NC, 1910)

a retort of disgust on receiving bad news or unpleasant orders
(F) réplique de dégoût en apprenant de mauvaises nouvelles ou en recevant des instructions désagréables

(D) Antwort des Ueberdrusses beim

Erfahren von schlechten Nachrichten oder beim Erhalten von unangenehmen

Anweisungen

to knock s.o. for a row of Chinese pagodas (NC, 1930)

1. to knock s.o. out 2. to make a great impression upon s.o.

(F) 1. assommer qqn 2. faire grosse impression sur qqn

(D) 1. jemanden übel zurichten 2. einen

grossen Eindruck auf jemanden machen

\section{COLOMBIA}

Colombian/Columbian gold (US, NC, 20)

(cf. Acapulco)

Colombian roulette (US, NC, 20)

cocaine traffic

(F) trafic de cocaine

(D) Kokainschwarzhandel

Colombian red (US, NC, 20)

(cf. Acapulco)

CONGO

Congo hash (US, NC, 20)

strong marihuana

(F) marijuana forte

(D) starke Marihuana

Congo mataby (US, NC, 20)

(cf. Acapulco)

\section{CONNAUGHT}

go to Hell or Connaught! (NC, 1654)

(cf. go to Bath!)

what the Connaught men shot at (NC, 1883) nothing at all

(F) rien du tout

(D) gar nichts

CORINTH

Corinth (NC, 17-19)

brothel

(F) maison close

(D) Bordell

Corinthian (NC, ob.)

1. man about town 2. fast liver

(F) 1. homme du monde 2. viveur, noceur

(D) 1. Weltmann 2. Lebemann

\section{CORK}

Earl of Cork (RS, 1830)

(cf. Duke of York $1 \&$ 2)

\section{CORNWALL}

to send (a man) to Cornwall without a boat

(NC, 1565-1830)

to cuckold a man 
(F) cocufier (un homme); envoyer en Cornouailles (NC, 20)

(D) zum Hahnrei machen

Cornish duck (NC, 1865, ob.)

pilchard

(F) pilchard

(D) Pilchard

Cornish hug

pretended friendship

(F) feinte manifestation d'amitié

(D) erheuchelte Freundschaftsbezeugung

\section{COVENT GARDEN}

Covent Garden (RS, 1857)

farthing

(F) quart de penny

(D) Viertelpenny

Covent Garden lady (1800)/nun (18-19)

prostitute

(F) prostituée

(D) Prostituierte

to break one's shins against Covent Garden rails

(NC, 18-19)

to catch a venereal disease

( $F)$ contracter une maladie vénérienne

(D) sich eine Geschlechtskrankheit zuziehen

\section{COVENTRY}

he has gone to Coventry (19-20)

he doesn't speak to me

(F) il ne me parle plus, il me bat froid

(D) er zeigt mir die kalte Schulter

to send S.o. to Coventry (18-20)

to ignore s.o. socially

(F) ignorer socialement qqn, ostraciser

(D) jemandem die kalte Schulter zeigen

\section{DALMATIA}

Dalmatian pudding (NC, 20)

currant-pudding

(F) pudding aux raisins

(D) Rosinenpudding

\section{DELAWARE}

square frome Delaware (US, NC)

old-fashioned person

(F) personne vieux jeu

DELHI

(D) altmodischer Mensch

Delhi belly (US, NC, 20)

(cf. Aztec two-step)

\section{DENMARK}

something is rotten in the kingdom of Denmark (17)

there's sthg shady in that business

(F) il y a quelque chose de louche dans cette affaire; il y a qqch de pourri dans le royaume de Danemark (17)
(D) etwas stimmt nicht in diesem Geschäft; etwas ist faul im Staate Dänemark (1850)

\section{DERBY}

Derby brights (RS, 20)

lights
(F) lumières
(D) Lichter

\section{DERBYSHIRE}

Derbyshire born and bred (NC, 19) boorish

(F) lourdaud

(D) Flegel; Kaffer (NC, 18)

\section{DOVER}

Dover harbour (RS, 20)

barber

(F) barbier, coiffeur (hommes)

(D) Herrenfriseur

Chatham and Dover (RS, 1909)

(cf. Chatham)

\section{DUBLIN}

Dublin dissector (NC, 1840-1900)

cudgel

(F) gourdin, trique

(D) Knüttel, Prügel

Dublin tricks (RS, 1860-1940)

bricks

(F) briques

(D) Ziegelsteine

to take the Dublin packet (NC, 1859, ob.)

to run away

(F) se sauver

(D) davonlaufen

to tip s.o. the Dublin packet (NC, 1852-1900)

to avoid s.o., to give s.o. the slip

(F) éviter qqn, fausser compagnie à qqn

(D) jemanden offen meiden, jemandem davonlaufen

\section{DURHAM}

Durham (US, NC, 20)

(cf. Burma shave)

Durham man (NC, 18-19)

knock-kneed man

(F) individu aux genoux cagneux

(D) krummbeiniger $M$ ensch

\section{DUTCH}

(cf. Netherlands)

\section{EGYPT}

Egypt (NC, 20, ob.)

bread

(F) pain

(D) Brot

corn in Egypt (1830)

plenty of food

(F) abondance de nourriture 
(D) U eberfluss an Lebensmittel

to go to Egypt (US, NC, 1910)

(cf. to give the Chinaman a music lesson)

to sigh for the fleshpots of Egypt

to regret a former situation

(F) regretter une situation antérieure;

regretter les oignons d'Égypte

(D) sich nach einer früheren Lage zurücksehnen; sich nach den Fleischtöpfen

Aegyptens sehnen

Egyptian (16)

(cf. Bohemian 1)

Egyptian Hall (RS, 1859)

ball

(F) bal

(D) Ball

to show an Egyptian medal (NC, 1884, ob.)

(cf. Abyssinian medal)

\section{ENGLAND}

England's umbrella (NC, 1923)

Ireland

(F) I'Irlande

(D) Irland

map of England (NC, 19-20)

sperm stains on the sheets

(F) taches de sperme sur les draps; carte de France (NC)

(D) Samenflecken auf dem Betttuch

English burgundy (NC, 18-19)

(cf. British champagne)

English pluck (NC, 1909, ob.)

money

(F) argent; ; japonais (NC, 1926), monacos

(NC, 1842-1931)

(D) Geld

in plain English (17)

frankly, bluntly

(F) clairement, sans ménagements; en bon français

(D) geradeheraus, unumwunden; auf gut Deutsch (16)

don't you understand plain English? (17)

won't you listen?

(F) tu ne veux pas écouter?; tu ne com-

prends pas/plus le français?

(D) willst du nicht gehorchen ?; du verstehst

wohl nicht mehr Deutsch/wohl kein Deutsch mehr?

\section{EPSOM}

Epsom races (RS)

1. braces (1857) 2. faces (1850-1900)

(F) 1 . bretelles 2 . visages

(D) 1. Hosenträger 2. Gesichter

\section{ESKIMO}

Eskimo kisser (NC, 20) nose

(F) nez

(D) Nase

Eskimo pie (NC, 20)

choc ice

(F) chocolat glacé, esquimau (NC, 20)

(D) Eisschoko

\section{ESSEX}

Essex calf (NC, 1570, ob.)

a native of Essex

(F) natif de l'Essex

(D) Eingeborener vom Essex

Essex lion (NC, 1620, ob.)

calf

(F) veau

(D) Kalb

Essex stiles (NC, 17-19)

ditches

(F) fossés, douves

(D) (Wasser) Graben

\section{ETHIOPIA}

Ethiopian (16)

negro

(F) nègre

(D) Neger

Ethiopian gallery/paradise (NC, 19-20) upper gallery in a theatre

(F) galerie supérieure d'un théâtre

(D) oberste Galerie eines Theaters

\section{EUROPE}

to go to Europe with Ralph and Earl in a Buick

(US, NC, 20)

to vomit

(F) vomir

(D) erbrechen; nach Speyer appellieren (NC, 19)

\section{EVERTON}

Everton toffee (RS, 1857)

coffee

(F) café

(D) Kaffee

\section{FIFE}

Duchess of Fife (RS, 19)

wife

(F) épouse

(D) Ehefrau

Duke of Fife (RS, 19-20)

knife

(F) couteau

(D) Messer

\section{FLANDERS}

Flanders fortune (NC, 17-18)

small fortune

(F) petite fortune

(D) kleines Vermögen 
Flanders piece (NC, 17-18)

coarse picture that looks fair at a distance

(F) peinture grossière qui ne paraît bien que de loin

(D) grobes Gemälde, dass nur aus der Ferne gut aussieht

Flemish account (NC, 1660-1874)

bad account

(F) compte erroné

(D) irrtümliche Rechnung

\section{FLORIDA}

Florida snow (US, NC, 20)

\section{FRANCE}

(cf. Bolivian marching powder)

France and Spain (RS, 19-20)

rain

(F) pluie

(D) Regen

Isle of France (RS, 1859, ob.)

dance

(F) danse

(D) Tanz

South of France (RS, 19-20)

(cf. ci-dessus)

French

1. fellation (NC, 1918), 2. dirty talk, swearing (US, NC, 1957)

(F) 1. fellation 2. gros mots, jurons

(D) 1. Fellatio 2. Schimpfreden, Flüche

French ache/disease/fever/goods/ gout/measles/ marbles/mole/pox (NC, 17-19)

syphilis

(F) syphilis; mal français/espagnol/de Naples

(17)

(D) Syphilis; Franzosenkrankheit

French article (NC, 1821)/cream (NC, 1788)/

elixir (NC, 1860)/lace (NC, 1821)

brandy

(F) cognac

(D) Weinbrand

French blue (NC, 20)

a mixture of amphetamines and barbiturates

(F) mélange d'amphétamines et de barbituriques

(D) M ischung von Amphetaminen und Barbituraten

French curves (US, NC, 20)

curvaceous body (of woman)

(F) corps harmonieux (en parlant d'une femme)

(D) kurvenreicher Körper (einer Frau)

French harp (US, NC)

harmonica

(F) harmonica

(D) Harmonika
French kiss

1. cf. French 1 (US, NC, 20)

2. tongue kiss (NC, 1923)

(F) baiser lingual; baiser florentin

(D) heisser Kuss

French letter (NC, 1870)

(cf. American letter)

French pie mutton stew

(F) ragoût de mouton à l'irlandaise

(D) Schafsragout auf irische Art

French pig (NC, 1790)

venereal bubo

(F) bubon vénérien

(D) Syphilisbeule

French prints (NC, 1850, ob.)

obscene pictures

(F) images ou photos obscènes

(D) Schmutzbilder

French safe (NC, 1910)

(cf. American letter)

French tricks (NC, 19-20)

sexual aberrations

(F) anomalies sexuelles (cunnilingus,

fellation)

(D) Sexualabweichungen

French walk (NC, 20)

forceful ejection

(F) expulsion brutale de qqn

(D) brutales Herausschmeissen

French way (US, NC, 1918)

(cf. French tricks)

pardon my French!

excuse the coarse language

(F) excusez la grossièreté de mon langage

(D) entschuldigen Sie meine gemeine

Ausdrucksweise

pedlar's French

1. cant (NC, 1530-20, ob.) 2. gibberish (NC, 17-19)

(F) 1. argot du milieu; breton (NC, 1795) 2.

charabia; chinois, grec, hébreu, iroquois

(D) 1. Gaunersprache 2. unverständliche

Sprache, Kauderwelsch

to drive French horses (19)

(cf. to go to Europe with Ralph and Earl in a Buick)

not to give a French-fried fuck (US, NC, 20)

not to care at all

(F) s'en moquer complètement

(D) sich gar nichts aus etwas machen

to take French leave (1770)

to depart unobtrusively, without intimation

(F) partir subrepticement; filer à l'anglaise (1890) 
(D) heimlich, ohne sich zu verabschieden davongehen; sich französisch verabschieden

\section{GALILEE}

\section{Galilee (US, 1920, ob.) \\ the Southern States \\ (F) les États du Sud \\ (D) die südlichen Staaten \\ GEORGIA \\ Georgia peach (US, NC) \\ pretty girl \\ (F) jolie fille \\ (D) hübsches $M$ ädchen}

\section{GERMANY}

made in Germany (19-20)

cheap, low-grade

(F) médiocre, sans valeur

(D) minderwertig, billig

German bands (RS, 19-20, ob.)

hands

(F) mains

(D) Hände

German duck (NC, 1860, ob.)

bed bug

(F) punaise

(D) Floh, Wanze

German flutes (RS, 1857-1914)

boots

(F) bottines, chaussures

(D) Schuhe

German goiter (US, NC)

beerdrinker's belly

(F) gros ventre (de buveur de bière) ; ventre

à la Suisse (17)

(D) Bierbauch

German gospel (NC, 1897)

arrogant bragging

(F) vantardises orgueilleuses

(D) hochmutige Prahlerei

to talk German (US, NC, 20)

to break wind

(F) péter

(D) furzen

\section{GIBRALTAR}

Gilbraltar (US, NC, 20)

(cf. Bolivian marching powder)

\section{GLASGOW}

Glasgow boat (RS, 19-20)

coat

(F) manteau, veston

(D) M antel, Jacke

Glasgow magistrate (NC, 1830, ob.)

high-quality herring

(F) hareng de qualité supérieure

(D) Hering erster Qualität
Glasgow rangers (RS, 19-20), glasgows

strangers, unknown people

(F) étrangers, inconnus

(D) Fremder, Unbekannter

\section{GOMORRAH}

Gomorrah to you! (NC, 1900-14)

good morning to you!

$(\mathrm{F})$ bonjour à vous!

(D) guten Tag!

GORGONZOLA

gorgonzola (NC, 20)

excellent, very well

(F) excellent, très bien; de Tolède (1872)

(D) erstklassig, prima

\section{GOTHAM}

wise man of Gotham (1694, ob.)

numbskull

(F) nigaud; Jean Ridou, marguillier de Saint-

Cloud (1640, ob.)

(D) Einfaltspinsel

\section{GOTHS}

Goth (1663)

uncouth person

(F) individu grossier, non civilisé, ignorant; ostrogoth (1690)

(D) roher, ungebildeter M ensch; Kaffer ( $\mathrm{NC}$, 18)

\section{GRAVESEND}

Gravesend bus (NC, 1880-1920)

hearse

(F) corbillard

(D) Leichenwagen

Gravesend sweetmeat (NC, 1860-1920)

shrimps

(F) crevettes

(D) Krabben

\section{GREECE}

Grecian

1. cf. Corinthian 2 (1818-60)

2. Irishman (NC, 1853, ob.)

(F) Irlandais

(D) Ire

Grecian accent (NC, 1850-1930)

Irish brogue

(F) accent irlandais

(D) irischer Akzent

Grecian gift

treacherous gift

(F) cadeau empoisonné

(D) betrügerisches Geschenk; ein Geschenk

Greek wie das trojanische Pferd

1. cf. Grecian 2 (NC, 1823, ob.)

2. cf. pedlar's French (1600) 
3. card-sharper, cheat (16-19)

(F) tricheur (au jeu); grec (1721)

(D) Falschspieler

Greek fashion/way, the (US, NC, 1940)

(cf. Bucklebury)

Greek fire (NC, 1889, ob.)

bad whisky

(F) mauvais whisky

(D) schlechter Whisky

Greek gift

(cf. Grecian gift)

Greek love (US, NC, 20)

(cf. Bucklebury)

at/on the Greek calends (16)

never

(F) jamais; aux calendes grecques (16)

(D) niemals

it's a case of Greek meeting Greek

forces are equal

(F) la lutte est égale

(D) es ist ein Fall von gleichen Kräften

it's all Greek to me (17-20)

I don't understand a word

(F) je n'y comprends rien; c'est de l'allemand/du haut-allemand (17), de l'auvergnat (NC, 20)/du chinois (1790)/du grec (17)/de I'hébreu (1530)/de l'iroquois (19) pour moi

(D) ich verstehe kein Wort; das sind mir böhmische Dörfer (16), das ist chinesisch für mich, das sind mir spanische Dörfer (18) merry as a Greek (US, NC)

(cf. unable to say British Constitution)

merry Greek (17, ob.)

(cf. Corinthian 2)

when Greek meets Greek then comes the tug of war

(cf. it's a case of Greek meeting Greek)

\section{GREENLAND}

to come from Greenland (NC, 1838, ob.)

to be unexperienced, guileless

(F) être sans expérience, naïf

(D) arglos, erfahrungslos sein

Greenlander

1. cf. Grecian 2 (NC, 1874, ob.)

2. artless person (NC, 1840, ob.) 3. beginner

(NC, 1840, ob.)

(F) 2. naïf 3. novice

(D) 2. argloser Mensch 3. Anfänger

\section{GRETNA GREEN}

Gretna Green marriage

marriage without parents' consent

(F) mariage sans autorisation parentale

(D) Heirat ohne elterliches Erlaubnis

\section{HALIFAX}

from Hell, Hull and Halifax, Good Lord, deliver us! (NC, 16-20)

deliver us from evil

(F) sauvez-nous du mal

(D) erretten Sie uns vom Bösen

go to Halifax! (NC, 17-20)

(cf. go to Bath !)

\section{HAMBURG}

Hamburg (NC, 19-20, ob.)

false rumour

(F) fausse rumeur

(D) falsche Nachricht

hamburger (US, NC)

1. of. China chin 2. tramp

3. minced meat sandwich

(F) 2. vagabond, clochard 3. sandwich de viande hachée

(D) 2. Bummler 3. Brötchen mit Hackfleisch

to make hamburger of s.o. (US, NC)

to thrash severely

(F) battre qqn violemment; péter le

maroquin à qqn (17)

(D) jemanden durchprügeln

HAMPSTEAD

Hampstead donkey (NC, 1865-1900)

(cf. Arkansas lizard)

H ampstead Heath (RS, 1880)

teeth

(F) dents

(D) Zähne

\section{HAMPTON}

Hampton wick (RS, 19-20)

prick, penis

(F) pénis; cure-dents d'Auvergne (NC, 17), chinois (NC, 1920)

(D) Penis

\section{HARWICH}

they're all up at (old) Harwich (NC, 1923)

they are in a pretty mess

(F) ils sont dans de beaux draps

(D) sie sitzen in der Patsche

\section{HASTINGS}

to be none of the Hastings sort (16-19)

to be a slowcoach

(F) être lent, indolent; être de Lagny, n'avoir pas de hâte (1640, ob.)

(D) träge, lässig sein

\section{HEBREWS}

Hebrew (NC, 1705)

(cf. Greek 3)

that's all Hebrew to me! (NC, 1705)

(cf. it's all Greek to me!) 


\section{HEREFORDSHIRE}

Herefordshire weed (NC, 1860)

oak

(F) chêne

(D) Eiche

\section{HERTFORDSHIRE}

Hertfordshire kindness (NC, 1660-1830)

recognition of favours received

(F) reconnaissance de faveurs reçues

(D) Anerkennung erhaltener Begünstigungen

\section{HIGHLAND}

Highland frisky (RS, 1870-1950) whisky

dona Highland-flinger (RS, 1909)

music-hall singer

(F) chanteuse de music-hall

(D) Variétésängerin

\section{HITTITES}

Hittite (NC, 1820-1910)

professional boxer

(F) boxeur professionnel

(D) Profi-Boxer

\section{HOLLAND}

the Dutch have taken Holland (17-18)

that's stale news

(F) c'est de l'histoire ancienne

(D) das ist eine altbackene Nachricht

\section{HONG KONG}

Hong Kong dog (NC, 20)

(cf. Aztec two-step)

go to Hong Kong! (NC, 19-20)

(cf. go to Bath!)

HOTTENTOTS

Hottentot (NC, 19)

(cf. Bœotian)

HOUNSLOW

Hounslow Heath (RS, 1857)

(cf. Hampstead Heath)

\section{HULL}

from Hell, Hull and Halifax, Good Lord, deliver us!

\section{HUNS}

(cf. Halifax)

Hun (1914-18-1929)

1. German soldier 2. German 3. despicable person

(F) 1. soldat allemand 2. Allemand 3. personnage méprisable

(D) 1. deutscher Soldat 2. Deutscher 3. verächtlicher M ensch

\section{HUNTINGDON}

Huntingdon sturgeon (NC, 1667-1830)

native or resident of Huntingdon

(F) natif ou habitant de Huntingdon
(D) Eingeborener bezw. Einwohner von

Huntingdon

\section{ILLINOIS}

Illinois green (US, NC, 20)

(cf. Acapulco)

\section{INCA}

Inca message (US, NC, 20)

(cf. Bolivian marching powder)

\section{INDIA}

India

1. cf. Acapulco 2. cf. berkeley 1

India rubber

eraser

(F) gomme à effacer

(D) Radiergummi

Indian bow paralysis

paralysis of thyroarytenoid muscle

(F) paralysie du muscle thyroaryténoïde

(D) Lähmung des Thyreoarytänoidmuskels Indian gift

a present in exchange for another

(F) cadeau en échange d'un autre

(D) Geschenk in Erwartung eines

Gegengeschenks; Indianergeschenk

Indian giver

person who takes back his present or expects one in exchange

(F) personne qui reprend son cadeau ou qui en attend un en échange

(D) jemand der sein Geschenk zurücknimmt bezw. ein Gegengeschenk erwartet

Indian hay/hemp (US, NC, 1934)

(cf. Acapulco)

Indian ink

black drawing ink

(F) encre de Chine

(D) chinesische Tusche

Indian powwow

boisterous meeting

(F) réunion bruyante

(D) laute Versammlung

Indian wipe (NC, 1790-1840)

silk handkerchief

(F) mouchoir de soie

(D) Seidentuch

all chiefs, no Indians! (NC, 19)

more bosses than workers

(F) il y a plus de chefs que d'exécutants;

c'est l'armée bolivienne! (NC, 20)

(D) hier ist jederman Chef!

honest Indian! (NC, 1880, ob.)

upon my word!

(F) parole d'honneur

(D) auf Ehrenwort! 
in Indian file

in a single file

(F) à la queue leu leu; en file indienne

(D) hintereinander, in Gänsemarsch

\section{IRELAND}

map of Ireland

(cf. map of England)

Irish

1. Irish whisky (NC, 1880) 2. anger (NC, 1870)

(F) 1. whisky irlandais 2. colère

(D) 1. irischer Whisky 2. Zorn

Irish apples (NC, 19)/apricots (NC, 1815)/

grapes (US, 19)/lemons (NC, 1815)

potatoes

(F) pommes de terre; canadas

(D) Kartoffeln ; preussische Beilage (NC, 20)

Irish banjo/fan (US, NC, 1930)/harp(NC, 1905)

shovel

(F) pelle

(D) Schaufel

Irish battleship/man-of-war (NC, 19-20, ob.)

barge

(F) chaland, péniche

(D) Zille, Punt

Irish buggy (US, NC, 1915)/local (US, NC, 1956) wheelbarrow

(F) brouette

(D) Schubkarre

Irish bull (NC, 1850)

(cf. Burma shave)

I rish button (US, NC, 20)

(cf. French pig)

Irish cherry (US, NC)

carrot

(F) carotte

(D) M öhre

Irish confetti (US, NC)

(cf. Dublin tricks)

I rish dividend (US, NC)

fine

(F) amende

(D) Geldstrafe

Irish draperies (NC, 1909)

cobwebs

(F) toiles d'araignées

(D) Spinnengewebe

Irish evidence (NC, 18-19)

false evidence

(F) faux témoignage

(D) Meineid

Irish fortune (NC, 19)

(cf. Cambridge fortune)

Irish horse (NC, 1887, ob.)

corned beef
(F) bœuf en conserve

(D) Büchsenfleisch

Irish kiss (US, NC, 19-20)

slap in the face

(F) gifle

(D) Ohrfeige

Irish mutton (US, NC, 1910)

(cf. French ache)

Irish promotion (NC, 1850-1910)

wage cut, reduction to a lower position

(F) réduction de salaire, rétrogradation

(D) Lohnverminderung, Rückschritt

Irish root (NC, 1830-1914)

(cf. Hampton wick)

Irish shave (US, NC, 1910)

defecation

(F) défécation

(D) Darmentleerung

Irish toothache

1. priapism (NC, 19-20, ob.)

2. pregnancy (NC, 1909)

(F) 1. priapisme 2. grossesse

(D) 1. Rutenkrampf 2. Schwangerschaft

Irish turkey (US, NC, 1935)

minced meat

(F) hachis de viande

(D) gehacktes Fleisch

Irish welcome (19-20)

standing invitation

(F) invitation permanente

(D) ständige Einladung

Irish whist (NC, 1859, ob.)

(cf. bike ride to Brighton)

as Irish as Paddy's/Pat's/Patrick's pig (NC, 1890)

staunch Irishman

(F) Irlandais bon teint

(D) echter Ire

lace-curtain Irish (US, NC)

well-to-do American of Irish descent

(F) Américain aisé d'origine irlandaise

(D) wohlhabender Amerikaner irischer

Abstammung

to get one's Irish up (NC, 1880)

to get angry

(F) se mettre en colère

(D) in Zorn geraten

to have danced at an Irish wedding (NC, 1840,

ob.)

to have bunged-up eyes

(F) avoir les yeux pochés

(D) um die Augen herum blau geschlagen

sein

to weep Irish (NC, 19-20)

to shed insincere tears 
(F) verser des larmes de crocodile

(D) Krokodilträne vergiessen

you're Irish! (NC, 19-20)

you're talking nonsense

(F) tu dis des sottises!

(D) du redest Unsinn!

Irishman, the (NC, 1905)

swindle

(F) escroquerie

(D) Schwindel

Irishman's hurricane (NC, 19-20, ob.) dead calm (at sea)

(F) calme plat (en mer)

(D) Windstille

Irishman's promotion/rise (NC, 1889)

(cf. Irish promotion)

to have an Irishman's dinner (NC, 19-20, ob.)

to go without eating

(F) se passer de manger

(D) nichts zu essen bekommen

smoked Irishman (US, NC, 20)

(cf. Ethiopian)

ISLE OF WIGHT

Isle of Wight $(\mathrm{RS}, 20)$

1. cf. unable to say British Constitution 2. right

(F) bien, parfait

(D) gut, richtig

\section{ITALY}

Italian letter (NC, 18-20)

(cf. American letter)

Italian manner (NC, 19-20)

(cf. Bucklebury)

Italian perfume (US, NC, 1910)

garlic

(F) ail

(D) Knoblauch

JAMAICA

old Jamaica (rum) (RS, 19-20)

(cf. Bengal blanket 1)

\section{JAPAN}

Japan (NC, 1915, ob.)

$$
\text { (cf. Egypt) }
$$

Japanese knife-trick (NC, 1885-1910)

to eat with one's knife

(F) manger avec son couteau

(D) mit dem Messer essen

\section{JAVA}

java (US, 1850)

(cf. Everton toffee)

\section{JERICHO}

Jericho (NC, 1840-1915)

toilet

(F) WC

(D) Klosett from Jericho to June (NC, 1835-1915)

a long distance

(F) une longue distance

(D) eine grosse Entfernung

go to Jericho! (NC, 1635)

(cf. go to Bath!)

to have been to Jericho (NC, 18-19)

(cf. he's been to Bungay fair and broken both his legs)

to send s. 0. to/to wish s.o. in Jericho (NC, 17)

to wish S.O. to hell

(F) envoyer qqn au diable; envoyer qqn à Cancale (17)/à Pampelune (17)/se faire voir chez les Grecs (NC, 20)

(D) jemanden zum Teufel wünschen ; jn. nach Buxtehude wünschen (NC, 1850)

\section{JERSEY}

Jersey Cities (RS, 1910)

(cf. berkeleys)

Jersey green (US, NC, 20)

(cf. Acapulco)

\section{JERUSALEM}

Jerusalem! (NC, 19-20)

exclamation of surprise

(F) exclamation de surprise

(D) Ausruf der U eberraschung; so was lebt

in China!

Jerusalem cuckoo

1. cf. Arcadian nightingale

2. mule (NC, 1917)

(F) mule

(D) M aulesel

Jerusalem parrot (NC, 1923)

(cf. German duck)

Jerusalem pony (NC, 1820, ob.)

(cf. Arcadian nightingale)

go to Jerusalem! (NC, 19-20)

(cf. go to Bath!)

Holy Jerusalem! (US, NC, 20)

an exclamation or swear-word

(F) exclamation ou juron

(D) Ausruf oder Fluch; Kruzitürken! (NC,

19)

to be going to Jerusalem (NC, 18-19)

(cf. he's been to Bungay fair and broken both his legs)

\section{JEWS}

Jew (1845)

hard bargainer, usurer

(F) individu dur en affaires, usurier ; juif

(17)

(D) einer der geschäftlich hart ist; Jude

jew, to (1845)

to cheat, swindle

(F) tromper; tricher 
(D) betrügen

Jew butter (US, NC, 19-20)

chicken fat

(F) graisse de poulet

(D) Hühnerfett

Jew-canoe (US, NC, 1940)

big car

(F) grosse voiture

(D) grosses Auto

Jew chum (NC, 20)

(cf. hamburger 2)

jew down, to (19)

to bargain

(F) marchander

(D) feilschen

Jew's compliment (NC, 1850, ob.)

a large penis but no money

(F) un pénis imposant mais aucun argent

(D) ein grosser Penis, aber kein Geld

Jew's Rolls-Royce (NC, 1938)

Jaguar motor-car

(F) voiture Jaguar

(D) Jaguarwagen

Jew York (US, NC, 20)

New York

as thick as two Jews on a pay-day (NC, 1887)

very intimate

(F) intimement liés

(D) innig verbunden

tell that to the Jews!

I don't believe you

(F) à d'autres!

(D) das macht anderen weis!

worth a Jew's eye (NC, 16-20, ob.)

very precious

(F) de grande valeur, précieux

(D) sehr wertvoll

Jewish flag (US, NC, 1929)

dollar note

(F) billet d'un dollar

(D) Dollarnote

Jewish nightcap (NC, 19-20)

foreskin

(F) prépuce

(D) Vorhaut

Jewish pianola (NC, 1925)

cash-register

(F) caisse enregistreuse

(D) Registrierkasse

\section{JUTLAND}

Jutland (NC, 18)

(cf. Brunswick 1)

\section{KAFFIRS}

Kaffir (NC, 1860-1910)

procurer
(F) proxénète

(D) Zuhälter

\section{KALMUKS}

Kalmuk idiocy

(cf. M ongolian idiocy)

\section{KANSAS}

Kansas neck blister (US, NC)

(cf. Arkansas toothpick)

\section{KARACHI}

Karachi (US, NC, 20)

heroin + depressant

(F) héroïne + dépresseur

(D) Heroin + Depressor

\section{KENT}

Duke of Kent (RS, 1932)

(cf. Burton-on-Trent)

Kentish cousin distant relation

(F) parent éloigné; parent à la mode de Bretagne (17)

(D) weitläufiger Verwandter

Kentish fire (1830, ob.)

salvo of applause

(F) applaudissements prolongés

(D) dauerndes $\mathrm{H}$ ändeklatschen

Kentish long-tail (NC, 13-20)

native or resident of Kent

(F) natif ou habitant du Kent

(D) Eingeborener bezw. Einwohner vom

Kent

\section{KENTUCKY}

Kentucky dew (US, NC, 20)

illicit whisky

(F) whisky clandestin

(D) Schwarzwhisky

Kentucky fried (US, NC, 20)

(cf. unable to say British Constitution)

Kentucky oysters (US, NC)

chitterlings

(F) tripes, andouillette

(D) Kaldaunen

Kentucky pill (US, NC, 20) bullet

\section{KERRY}

(F) projectile d'arme à feu

(D) Kugel

Kerry witness (NC, 1825, ob.) one who will swear to anything

(F) individu prêt à jurer de tout

(D) einer der bereit ist alles zu schwören

\section{KHYBER PASS}

Khyber Pass

1. cf. Brunswick 1 (RS, 19-20)

2. glass (RS, 1885)

$(\mathrm{F})$ verre 
(D) Glas

\section{KILKENNY}

Kilkenny (RS, 1870)

(cf. Abergavenny)

to fight like Kilkenny cats (NC, 19-20)

to fight savagely

(F) se battre sans ménagement

(D) sich bis aufs Blut bekämpfen

\section{KILLARNEY}

Lakes of Killarney (RS, 20)

barmy, mad

(F) fou

(D) verrückt

\section{LANCASHIRE}

Lancashire lass (RS, 1880)

(cf. Khyber Pass 2)

Lancashire lasses (RS, 1959)

spectacles

(F) lunettes

(D) Brille

\section{LAPLAND}

Lapland (NC, 1820)

1. cf. berkeley 1

2. womenfolk

(F) la gent féminine

(D) Weibervolk

\section{LESBOS}

lesbian (1900)

female homosexual

(F) homosexuelle; lesbienne

(D) Tribade; Lesbierin

\section{LIMBURG}

that's the Limburger ! (NC, 19-20)

(cf. that's the Cheshire!)

\section{LINCOLN}

Lincoln's Inn (RS, 19-20)

5 -shilling piece

(F) pièce de 5 shillings

(D) Fünfschillingstück

\section{LINCOLNSHIRE}

Lincolnshire yellow-belly (NC, 18-20)

native or resident of Lincolnshire

(F) natif ou habitant du Lincolnshire

(D) Eingeborener bezw. Einwohner vom Lincolnshire

\section{LIVERPOOL}

Liverpool kiss (US, NC)

blow on the mouth

(F) coup sur la bouche

(D) Schlag auf den Mund

Liverpool wash (US, NC)

wash limited to the upper part of the body

(F) toilette de la partie supérieure du corps seulement

(D) sich nur den oberen Körperteil waschen

\section{LOMBARDY}

lombard (1709, ob.)

money-changer/-lender

(F) changeur, prêteur ; lombard

(D) Geldwechsler/-verleiher; Lombarde

Lombard fever (NC, 1678-1870)

laziness, idleness

(F) paresse, oisiveté

(D) Faulenzerei, M üssiggang

in Lombard Street (NC, 1810-60)

in prison

(F) en prison

(D) im Gefängnis; hinter schwedischen

Gardinen (NC, 19)

it's Lombard Street to a Brummagem sixpence

(NC, 1826, ob.)

(cf. it's Chelsea College to a sentry-box)

\section{LONDON}

London fog (RS, 19-20)

dog

(F) chien

(D) Hund

London ivy (NC, 1852-1920)/particular (NC, 1852)

thick fog

(F) épais brouillard (Iondonien)

(D) dichter (Londener) Nebel

London milk (NC, 1880)

gin

(F) gin

(D) Gin

at number one London (NC, 19)

menstruation

(F) menstruation; débarquement des Anglais

(NC, 1948)

(D) menstruierend

to agree like the clocks of London (NC, 16-18)

to disagree on everything

(F) être en désaccord sur tout

(D) völlig uneinig sein

to put/show the best side to London (NC, 1873) to present oneself in one's best aspect

(F) se montrer sous son plus beau jour

(D) sich im schönsten Lichte zeigen

\section{LONDONDERRY}

Londonderry (RS, 20)

(cf. Bristol milk)

\section{MAIDSTONE}

Maidstone jailer (RS, 1857, ob.)

tailor

(F) tailleur

(D) Schneider

MALTA

Malta dog (NC, 19-20)

(cf. Aztec two-step) 
M altese lace (NC, 1905)

frayed edges of trousers, shirt-cuffs, etc.

(F) bords effilochés de pantalons, manchettes, etc.

(D) ausgefaserte Hosen- oder M anschettensäume

\section{MANCHESTER}

manchester (NC, 1812-1900)

tongue

(F) langue

(D) Zunge

$M$ anchester-bred (long in the arms and short in the head) (1869)

(cf. Bœotian)

M anchester Cities (RS, 19-20), manchesters (NC, 19-20)

(cf. berkeleys)

$M$ anchester silk (1850)

cotton

(F) coton

(D) Baumwolle

M anchester sovereign (NC, 1830, ob.)

shilling piece

(F) pièce d'un shilling

(D) Schillingstück

\section{MANH ATTAN}

Manhattan eel (US, NC, 20)

(cf. American letter)

M anhattan silver/white (US, NC, 20) (cf. Acapulco)

\section{MANILA}

Manila rope (US, NC)

cheap cigar

(F) cigare bon marché

(D) minderwertige Zigarre; Havanna mit

Plünneneinlage

\section{MARATH ON}

marathon (race) (1896)

race of $42,195 \mathrm{~km}$

(F) course à pied de $42,195 \mathrm{~km}$; marathon

(D) Lauf von 42,195 Km; M arathonlauf

marathon meeting (20)

very long meeting

(F) réunion de longue durée; séance

marathon

(D) langwierige Sitzung; M arathonsitzung

marathon speech (20)

long-winded speech

(F) discours interminable; marathon oratoire

(D) langatmige Rede; M arathonrede

\section{MARBLE ARCH}

Marble Arch (NC, 1840)

(cf. berkeley 1)

\section{MEMPHIS}

Memphis dominoes (US, NC, 20)

\section{(cf. African dominoes)}

\section{MESO POTAMIA}

the true M esopotamia ring (NC, 1880-1910)

high-sounding and incomprehensible way of expression

(F) parler ronflant et incompréhensible

(D) hochtrabende, unverständliche

Ausdrucksweise

\section{MEXICO}

Mexican (US, NC, 20)

1. cf. Acapulco 2. cf. made in Germany

M exican athlete (US, NC, ob.)

poor athlete

(F) athlète médiocre

(D) minderwertiger Athlet

M exican bankroll (US, NC, 20)

wad of counterfeit notes with only one

genuine on top

(F) liasse de faux billets avec un seul vrai par-dessus

(D) Bündel falscher Banknoten mit nur einer echten obenauf

M exican beer (US, NC, 20)

water

(F) eau

(D) Wasser

M exican breakfast (US, NC) a cigarette and a glass of water

(F) petit déjeuner réduit à une cigarette et

un verre d'eau

(D) Frühstück bestehend in eine Zigarette und ein Glas Wasser

Mexican brown (US, NC, 20)

(cf. Acapulco)

Mexican diamond (US, NC)

false brilliant

(F) faux brillant

(D) falscher Brillant

Mexican divorce/job (US)

swift divorce

(F) divorce rapide

(D) Schnellscheidung

M exican green (US, NC, 20)

(cf. Acapulco)

Mexican milk (US, NC, 19)

tequila
(F) téquila
(D) Tequila

Mexican mud (US, NC, 20)

(cf. China white)

Mexican promotion/raise (US, NC) promotion without pay rise

(F) avancement sans augmentation de salaire

(D) Beförderung ohne Gehaltserhöhung 
Mexican red (US, NC, 20)

(cf. Acapulco)

\section{MICHIGAN}

Michigan roll (US, NC)

(cf. Mexican bankroll)

MIDDLESEX

Middlesex clown (NC, 17-19)

native or inhabitant of Middlesex

(F) natif ou habitant du Middlesex

(D) Eingeborener bezw. Einwohner von M iddlesex

MIDLANDS

midlands (NC, 19)

(cf. berkeley 1)

\section{MILWAUKEE}

Milwaukee goiter (US, NC, 19)

(cf. German goiter)

\section{MISSISSIPI}

Mississipi marbles (US, NC)

(cf. African dominoes)

\section{MISSOURI}

Missouri toothpick (US, NC)

(cf. Arkansas toothpick)

from Missouri (US, NC)

incredulous, sceptical

(F) incrédule, sceptique

(D) ungläubig, skeptisch

I'm from Missouri ! (US, NC)

(cf. tell that to the Jews!)

\section{MONGOLIA}

M ongolian child (1892)

a child with trisomy 21

(F) enfant atteint de trisomie 21; mongol(ien) (1866)

(D) Kind mit Trisomie 21; M ongoloider

M ongolian idiocy, mongolism (1892)

Down syndrome

(F) syndrome de Down; mongolisme (1866)

(D) Downsyndrom; M ongolismus

\section{MOROCCO}

in M orocco (NC, 1850)

stark naked

(F) tout nu

(D) ganz nackt

\section{MOSCOW}

M oscow (NC, 20)

pawnshop

(F) mont-de-piété

(D) Pfandhaus; Lombard

gone to Moscow (NC, 20)

in pawn

(F) mis en gage

(D) verpfändet; (ein Gegenstand) lernt

hebräisch (NC, 1850)

\section{MUNSTER}

Munster heifer (NC, 1810-60)

thick-legged woman

(F) femme aux très grosses jambes

(D) dickbeinige Frau

Munster plums (NC, 1780, ob.)

(cf. Irish apples)

\section{NAPLES}

Neapolitan disease (NC, 16-17)/favour (NC, 1819)

(cf. French ache)

\section{NEBRASKA}

Nebraska sign (US, 20)

death

(F) signe de décès

(D) Todeszeichen

\section{NEEDHAM}

Needham (NC, 1570-1890)

poverty, misery

(F) pauvreté, misère

(D) Armut, Not

\section{NETHERLANDS, THE}

Netherlands, the (NC, 18-20, ob.)

the male or female genitals

(F) parties génitales de l'homme ou de la femme; Pays-Bas (NC, 1863, fém. seulement)

(D) die männlichen oder weiblichen

Genitalien

Dutch act/cure (US, NC, 1935)

suicide

(F) suicide

(D) Selbstmord

Dutch anchor (US, NC, 1860)

something important forgotten somewhere

on leaving

(F) chose importante oubliée quelque part

en quittant

(D) wichtige Sache, die man irgendwo beim

Abschied vergessen hat

Dutch auction/sale (NC, 1864)

mock auction or sale

(F) simulacre d'enchères

(D) Scheinversteigerung

Dutch bargain (NC, 17)

a bargain for only one of the parties

(F) affaire avantageuse pour l'une des parties

seulement

(D) Geschäft dass nur für eine Partei vorteilhaft ist

Dutch boy (US, NC, 20)

(cf. Brighton Pier 2)

Dutch cap (NC, 1925)

pessary

$(\mathrm{F})$ pessaire 
(D) Mutterzäpfchen

Dutch cheese (NC, 1882-1915)

bald-head

(F) chauve

(D) Kahlkopf

Dutch clock, old Dutch clock (NC, 19, ob.)

(cf. Duchess of Fife)

Dutch comfort (NC, 1787)/consolation (NC, 19)

a consolation which is none

(F) consolation qui n'en est pas une

(D) Trost der kein ist

Dutch concert (NC, 1780)/medley

(NC, 19-20, ob.)

caterwauling

(F) cacophonie

(D) Katzenmusik

Dutch courage (17)

courage from drinking

(F) bravoure après boire

(D) angetrunkener Mut

Dutch defence

surrender

(F) capitulation

(D) Uebergabe

Dutch kiss (NC, 1935)

sexual intimacies

(F) privautés sexuelles

(D) Geschlechtsverkehr

Dutch luck (US, NC)

undeserved luck

(F) chance imméritée

(D) unverdientes Glück

Dutch nightingale (NC, 1769, ob.)

(cf. Cambridgeshire nightingale)

Dutch oven (NC, 1923)

the mouth

(F) la bouche

(D) der Mund

Dutch party (20)/treat (1875)

(cf. Chinaman's shout)

Dutch pegs (RS, 1923)

legs

(F) jambes

(D) Beine

Dutch pink (NC, 1853)

blood

(F) sang

(D) Blut

Dutch praise (US, NC)

blame under the appearance of praise

(F) blâme sous I'apparence de louanges

(D) Tadel unter dem Schein des Lobes

Dutch reckoning (NC, 1690-1800)

lump account
(F) compte non détaillé

(D) Gesamtrechnung

Dutch widow (NC, 1600-1750)

(cf. Covent Garden lady)

Dutch wife (NC, 1880, ob.)

bolster

(F) polochon, traversin

(D) Kissen

big Dutch (man) (US, NC)

boor

(F) gros lourdaud, rustre

(D) Flegel, Grobian; Kaffer (NC, 18), Kanake

(NC, 1850)

double Dutch (NC, 1860)

(cf. Greek 2)

double Dutch coiled against the sun (NC, 1818)

(cf. Burma shave)

in Dutch (US, NC, 20)

pregnant

( $F$ ) enceinte

(D) schwanger; auf der Wartburg (sitzen)

(NC, 1900)

it's all Dutch to me! (NC, 19)

(cf. it's all Greek to me!)

my old Dutch (NC, 1855)

my wife

(F) ma femme

(D) meine Frau

that beats the Dutch! (NC, 19-20)

that's incredible!; that's the limit !

(F) c'est inouï !; il ne manquait plus que ça!

(D) das ist unerhört!; das haut den stärksten

Eskimo vom Schlitten! (NC, 1930)

that's a Dutch reckoning

I've been fleeced

(F) c'est le coup de fusil ; c'est la forêt de

Bondy! (18-19)

(D) das ist eine richtige Prellerei!

the Dutch have taken Holland

(cf. Holland)

to be in Dutch (NC, 1935)

(cf. to be all up in Harwich)

to be in Dutch with s.o.(NC, 1925)

to be in disfavour with S.o.

(F) être mal vu de qqn

(D) bei jemandem unten durchsein

to do a Dutch (NC, 1870, ob.)

(cf. to take the Dublin packet)

to do/pull the Dutch act (NC, 1935)

to commit suicide

(F) se suicider

(D) sich das Leben nehmen

to eat/lunch in Dutch street (NC, 19-20)

to pay each one's own bill 
(F) payer chacun sa note; faire une anglaise (1867)

(D) jeder für sich bezahlen

to go Dutch (NC, 1914)

(cf. ci-dessus)

to take Dutch leave (US)

(cf. to take French leave)

to take part in a Dutch treat (NC, 19)

(cf. to eat in Dutch street)

to take the Dutch cure (NC, 20)

(cf. to do the Dutch act)

to talk double Dutch (NC, 1860)

to talk gibberish

(F) baragouiner; parler petit nègre (1857)

(D) Kauderwelsch reden

to talk to s.o. like a Dutch uncle (NC, 1830)

to lecture s.o.

(F) faire la morale à qqn

(D) jemanden M ohres lehren

Dutchman's anchor (US, NC, 1860)

(cf. Dutch anchor)

Dutchman's breeches (NC, 1867)

a little blue in a cloudy sky

(F) un peu de bleu dans un ciel de nuages

(D) ein bischen blau in einem Wolkenhimmel

Dutchman's drink (NC, 1860)

one that empties the glass

$(F)$ verre vidé d'un coup

(D) auf einen Zug ausgetrunkenes Glas

Dutchman's headache, the (NC, 1869-1920) drunkenness

(F) ivresse; palu breton (NC, 1940)

(D) Betrunkenheit

as drunk as a Dutchman (NC)

(cf. as merry as a Greek)

I'm a Dutchman! (NC, 1837)

(cf. tell that to the Jews!)

I'm a Dutchman if... (NC, 1850)

I'll be hanged if...

(F) je veux être pendu si...

(D) ich will Hans heissen, wenn...

to have a Dutchman's headache (NC, 19-20)

to feel sick the morning after

(F) se sentir mal au lendemain de libations trop abondantes

(D) einen Kater haben

\section{NEVADA}

Nevada divorce (US, NC)

(cf. Mexican divorce)

\section{NEWCASTLE}

Newcastle hospitality (NC, 19-20)

exaggerated kindness

(F) gentillesse exagérée

(D) übertriebene Liebenswürdigkeit to carry coals to Newcastle (16-20)

to do sthg useless or stupid

(F) faire qqch d'inutile ou de ridiculement superflu; vendre des coquilles à ceux qui viennent de Saint-M ichel (16)

(D) etwas Unnötiges, U eberflüssiges tun ;

Eulen nach Athen tragen

\section{NEWGATE}

Newgate hornpipe (NC, 19)

hanging

(F) pendaison

(D) Erhängen

Newgate gaol (RS, 19-20)

tale

(F) histoire, conte

(D) Geschichte, M ärchen

\section{NEWINGTON}

Newington butts (RS, 20), newingtons

guts

(F) entrailles

(D) Gedärm

\section{NEWMARKET}

a fine morning to catch herrings on N ewmarket

Heath (17-18)

a rainy day

(F) journée pluvieuse

(D) Regentag

\section{NEWTON}

Newton Heath (RS, 20)

(cf. Hampstead Heath)

\section{NEW YORK}

New York's finest (US, NC)

the City's police

(F) la police new-yorkaise

(D) die New Yorker Polizei

New York kiss-off (US, NC, 20)

(cf. California kiss-off)

New York nippers (RS, 20)

kippers

(F) harengs doux, kippers

(D) Kippers

\section{NIAGARA}

Niagara Falls (RS, 20)

1. stalls (1931) 2. testicles

(F) 1. fauteuils d'orchestre 2. testicules

(D) 1. Parkett 2. Hoden

\section{NORFOLK}

N orfolk capon (NC, 1780, ob.)

red herring

(F) hareng saur

(D) Pökelhering

Norfolk dumpling (NC, 17-20)/turkey (NC, 19-

20, ob.)

native or inhabitant of Norfolk

(F) natif ou résident du Norfolk 
(D) Eingeborener bezw. Einwohner vom Norfolk

\section{NORTH POLE}

North Pole (NC, 1870)

(cf. Brunswick 2)

\section{NORWAY}

N orway neck-cloth (NC, 1784-1830)

pillory

(F) pilori

(D) Schandpfahl

\section{NUREMBERG}

Nuremberg egg (NC, 16-18)

egg-shaped watch

(F) montre en forme d'œuf

(D) eiförmige Uhr

\section{ORINOKO}

Orinoko (RS, 1857, ob.)

poker

(F) poker

(D) Poker

\section{OXFORD}

oxford (NC, 1885-1914)

crown piece

(F) pièce d'une couronne

(D) Krone (Geldstück)

Oxford bags (1922)

wide-legged trousers

(F) pantalon bouffant

(D) Knickerbocker

Oxford scholar (RS)

1. collar (1870, ob.) 2. dollar (20)

(F) 1. col 2. dollar

(D) 1. Kragen 2. Dollar

\section{PALL MALL}

Pall Mall (RS, 19-1950)

girl

(F) jeune fille

(D) M ädchen

\section{PANAMA}

Panama gold/red (US, NC, 20)

(cf. Acapulco)

\section{PECKHAM}

Peckham Rye (RS, 20)

tie

(F) cravate

(D) Schlips

\section{PENNSYLVANIA}

Pennsylvania feathers (US, NC, 1915)

coal, coke

(F) charbon, coke

(D) Kohle, Koks

Pennsylvania salve (US, NC, 1920)

empty promises

(F) belles promesses, paroles en l'air

(D) leere Versprechungen

\section{PERSIA}

Persian brown/dust (US, NC, 20)

(cf. China white)

\section{PERU}

Peruvian flake (US, NC, 20)

(cf. Bolivian marching powder)

\section{PHILADELPHIA}

Philadelphia lawyer

1. smart attorney (US, NC, 1803; GB 1860-

1920) 2. shrewd person

(F) 1. avocat habile 2. personne rusée, astucieuse

(D) 1. geschickter Anwalt 2. Schlauberger

Philadelphia treat (US, NC)

(cf. Chinaman's shout)

enough to puzzle a Philadelphia lawyer

(cf. Chinese puzzle)

\section{PHILISTINES}

philistine

1. drunkard (17-18) 2. person deficient in liberal culture (1827)

(F) 1. ivrogne 2. personne peu cultivée;

philistin (1832)

(D) 1. Säufer; Kümmeltürke (NC) 2. M ensch mit sehr konservativen Ansichten ; Philister

(19)

PICCADILLY

Piccadilly (RS, 20)

silly

(F) stupide, niais

(D) dumm, blöd

\section{PITTSBURGH}

Pittsburgh of the South (US)

the City of Birmingham (Alabama)

(F) la ville de Birmingham

(D) die Stadt Birmingham

Pittsburgh of the West (US)

the city of Pueblo (Colorado)

(F) la ville de Pueblo

(D) die Stadt Pueblo

\section{PLYMOUTH}

Plymouth cloak (NC, 1608-1830)

(cf. Dublin dissector)

\section{POLAND}

Polish plait

plica polonica

(F) plique, trichome

(D) Weichselzopf

\section{PORTO}

portwine mark/stain (1700)

angioma

(F) angiome plan, tache de vin

(D) rotes Muttermal

PORT SAID

Port Said garter (NC, 1935-55) 


\author{
(cf. American letter) \\ PORTUGAL \\ Portuguese pump(ing) (NC, 1909) \\ masturbation \\ (F) masturbation \\ (D) Onanie \\ PRUSSIA \\ Prussian (NC, 20) \\ active sodomist \\ (F) pédéraste actif \\ (D) aktiver Päderast

\section{PUNJAB} \\ to have a Punjab head (NC, 1880) \\ to have a poor memory \\ (F) avoir mauvaise mémoire \\ (D) ein schlechtes Gedächtnis haben
}

\section{RAMSGATE}

Ramsgate Sands (RS, 20)

hands

(F) mains

(D) Hände

ROCHESTER

Rochester portion (NC, 17-19)

(cf. Cambridge fortune)

\section{ROME}

Rome was not built in a day (16)

patience is needed to achieve things

(F) les choses doivent se faire avec patience;

Paris/Rome ne s'est pas faite en un jour (17)

(D) es braucht Geduld um ein Ziel zu

erreichen; Rom ist nicht an einem Tage erbaut worden

all roads lead to Rome

there are many ways to achieve an end

(F) il y a de nombreux moyens d'arriver au même but; tous les chemins mènent à Rome (D) es gibt viele Mittel um ein Ziel zu erreichen ; alle Wege führen nach Rom

at Rome you must do as the Romans do one should comply with local usage

(F) il faut se conformer aux usages du pays;

à Rome, il faut vivre comme à Rome

(D) man soll sich nach den Gewohnheiten

des Landes richten; in Rom, tue wie Rom tut

gates (RS, 20)/pope (RS, 1859)/top (RS, 1857,

ob.) of Rome

home

(F) chez soi, home

(D) Heim

Roman penny (1860)

Peter's pence

(F) denier de saint Pierre (12)

(D) Peterspfennig

to have Roman hands (US, NC)

to caress women stealthily
(F) caresser les femmes subrepticement

(D) die Frauen heimlich streicheln

when at Rome do as the Romans do

(cf. at Rome you must do as the Romans do)

\section{ROMFORD}

Romford lion (NC, 18-19)

(cf. Essex lion)

to ride to Romford (NC, 1780-1830)

to get a new pair of breeches

(F) se procurer une nouvelle culotte (de cheval)

(D) sich eine neue Reithose anschaffen

you can ride to Romford on this knife (NC,

1705-1860)

that knife has no edge

(F) ce couteau est tout émoussé

(D) dieses Messer schneidet gar nicht; auf

diesem Messer kann man nach Rom reiten

\section{ROTTEN ROW}

Rotten Row (RS)

1. bow (1909) 2. blow (20)

(F) 1. arc 2. coup

(D) 1. Bogen 2. Schlag

RUSSIA

russia (NC, 1877, ob.)

wallet

(F) portefeuille

(D) Brieftasche

Russian duck (RS, 1923-59)

muck

(F) saletés, ordures

(D) Schweinerei

Russian socks (NC, 1935)

rags bound around the feet

(F) chiffons liés autour des pieds; chaussettes russes (20)

(D) Fusslappen

Russian Turk (RS, 1860-1910)

work

(F) travail

(D) Arbeit

black Russian (US, NC, 20)

hashish

(F) haschisch

(D) Haschisch

to have Russian fingers (US, NC)

(cf. to have Roman hands)

SAINT-MARTIN'S-LE-GRAND

Saint-M artin's-le-Grand (RS, 1857)

hand

(F) main

(D) Hand

SAMARIA

to be a good Samaritan (17)

to be compassionate 
(F) être secourable; jouer au bon Samaritain

(19)

(D) mitleidig sein; den barmherzigen

Samariter spielen

\section{SCARBOROUGH}

Scarborough warning (NC, 16-20, ob.)

too short a notice or no notice at all

(F) préavis trop court ou sans préavis

(D) kurzfristige or gar keine Kündigung

\section{SCOTLAND}

curse of Scotland (US, NC)

four of diamonds (cards)

(F) le quatre de carreau

(D) Karovier

Scotch (NC, 19-20)

miserly

(F) avare, regardant

(D) geizig, karg

Scotch answer/fashion (NC, 1834)

answering a question by another question

$(F)$ réponse à une question par une autre question

(D) Antwort auf eine Frage mit einer anderen Frage

Scotch casement (NC, 18-19)

(cf. Norway neck-cloth)

Scotch fiddle (NC, 1675, ob.)

the itch

(F) la gale

(D) Krätze

Scotch greys (NC, 19-20)

(cf. Arkansas lizard)

Scotch (NC, 1589)/Scottish (NC, 19-20) mist

1. cf. unable to say British Constitution 2.

soaking rain

(F) pluie continue

(D) Dauerregen

Scotch pegs

1. cf. Dutch pegs (RS, 1857)

2. eggs (RS, 20)

(F) œufs

(D) Eier

Scotch polo (US, NC)

golf

(F) golf

(D) Golfspiel

Scotch tea (NC, 1887)

whisky

(F) whisky

(D) Whisky

Scotch/Scottish warming-pan (NC, 1670-1880)

(cf. Dutch widow)

to make a Scotch bed (NC, 1918)

to fold blankets into the form of a sleepingbag
(F) plier les couvertures en forme de sac de couchage

(D) die Bettdecken sackweise falten

Scotchman (US, NC)

1. cf. Scotch

2. professional golf player

(F) joueur de golf professionnel

(D) Profi-Golfspieler

Scotchmen (NC, 1887)

(cf. Scotch greys)

\section{SEVILLE}

to learn manners in Seville (NC, 1923)

to learn civil manners

(F) apprendre les bonnes manières

(D) die guten U mgangsformen erlernen

\section{SHANGAI}

Shangai gentleman (NC, 1909)

quite the opposite of a gentleman

(F) tout le contraire d'un gentleman

(D) der Gegenteil eines Gentlemans

to shangai s.o. into doing sthy

to force s.o. to do sthy

(F) contraindre qqn à faire qqch

(D) einen zwingen etwas zu tun

\section{SHEFFIELD}

Sheffield handicap (NC, 20)

(cf. Irish shave)

\section{SIAM}

Siamese twins (1833)

inseparable friends

(F) ami(e)s inséparables; (être comme des)

frères/sœurs siamois(es) (1872)

(D) unzertrennliche Freunde

\section{SIBERIA}

to send to Siberia (US, NC, 20)

to move a troublesome policeman

(F) muter un policier gênant

(D) einen beschwerlichen Polizist versetzen

\section{SODOM}

Sodom (NC, 19)

the City of London

(F) la ville de Londres

(D) die Stadt London

it's a regular Sodom (17)

it's an immoral, corrupt place

(F) c'est un endroit immoral, corrompu ;

c'est Sodome et Gomorrhe

(D) es ist ein sittloses, verdorben Ort; es ist

ein wahres Sodom und Gomorra

sodomy (13-14)

(cf. Bucklebury)

\section{SPAIN}

castles in Spain (19-20)

daydream, idle fancy 
(F) projets chimériques; châteaux en Espagne (13)

(D) Luftschlösser

France and Spain (RS, 19-20)

(cf. France)

Spanish, the (NC, 1786, ob.)

(cf. English pluck)

Spanish athlete (US, NC, 20) braggart

(F) hâbleur; Gascon (17)

(D) Aufschneider

Spanish coin (NC, 1780-1850)/money (NC, 17-

18)

fair words and compliments

(F) (payer de) belles paroles

(D) (mit) leeren Worten (abspeisen)

Spanish faggot (NC, 1785-1850)

(cf. Bengal blanket 1 )

Spanish fly (US, NC, 20)

(cf. Bolivian marching powder)

Spanish football (NC, 1915)

venereal disease

(F) maladie vénérienne

(D) Geschlechtskrankheit

Spanish gout (NC, 17-19)/needle (NC, 19)/pox

(NC, 17-19)

(cf. French ache)

Spanish guitar (RS, 19-20)

cigar
(F) cigare
(D) Zigarre

Spanish letter (18-20)

(cf. American letter)

Spanish Main (RS, 20)

drain

(F) égout, rigole

(D) Abflussrohr

Spanish padlock (NC, 1786-1850)

chastity girdle

(F) ceinture de chasteté

(D) Keuschheitsgürtel

Spanish pennants (NC, 1905)

(cf. M altese lace)

Spanish trumpeter (NC, 1780-1850)

braying ass

(F) âne brayant

(D) schreiender Esel

Spanish walk (US, NC, 20)

(cf. French walk)

Spanish windlass (US, NC)

strait jacket

(F) camisole de force

(D) Zwangsjacke

to walk Spanish (NC, 19-20)

(cf. to do a Dutch)

\section{SUFFOLK}

Suffolk punch (NC, 1884)

native or inhabitant of Suffolk

(F) natif ou habitant du Suffolk

(D) Eingeborener bezw. Einwohner vom

Suffolk

Suffolk stiles (NC, 17-18)

(cf. Essex stiles)

\section{SWEDEN}

Little/New Sweden (US)

the State of Minnesota

(F) l'État du M innesota

(D) der Staat M innesota

Swede (US, NC)

1. clumsy fellow 2. bungled work

(F) 1. maladroit 2. travail mal fait

(D) 1. ungeschickter $M$ ensch 2. Pfuscharbeit

big Swede (US, NC)

(cf. big Dutch)

to set the swede down (NC, 1910)

to take a nap

(F) faire un petit somme

(D) ein Nickerchen machen

\section{SWITZERLAND}

Switzerland of America (US)

(cf. America)

Swiss admiral (1870, ob.)

one who has never seen the sea

(F) qui n'a jamais vu la mer; amiral suisse

(D) einer der nie in See gegangen ist

Holy Swiss cheese! (US, NC, 19-20)

(cf. Holy Jerusalem !)

\section{SYDNEY}

Sydney bird/duck (NC, 1850-90)

convict

(F) bagnard

(D) Sträfling

Sydney Harbour (RS, 19-20)

(cf. Dover Harbour)

Sydney-sider

1. cf. Sydney bird 2. native or inhabitant of Sydney (NC, 20)

(F) natif ou habitant de Sydney

(D) Eingeborener bezw. Einwohner von Sydney

it's either Sydney or the bush! (NC, 19-20)

a final choice or decision

(F) c'est I'un ou l'autre, il faut choisir !

(D) entscheide dich so oder so!

\section{TARTARY}

Tartar

1. thief, crook (NC, 1598-1780) 2. expert

(NC, 1780, ob.) 3. ruthless person (17)

(F) 1. voleur, escroc 2. expert 3. personnage brutal 
(D) 1. Dieb, Schwindler; Hanake (NC, 18) 2. Experte 3. brutaler Kerl

to catch a Tartar (17-20)

to come up against one's match

(F) trouver à qui parler

(D) seinen $M$ ann finden

to have caught a Tartar (17-20)

(cf. to be all up at Harwich)

\section{TEXAS}

Texas tea (US, NC, 20)

(cf. Acapulco)

tall Texas tale (US) incredible story

(F) histoire exagérée, incroyable; histoire de Gascon, gasconnade, histoire marseillaise (D) übertriebene, unglaubliche Geschichte

\section{THAILAND}

Thai stick (US, NC, 20)

(cf. African woodbine)

\section{TILBURY}

Tilbury Docks (RS, 19-20)

socks

(F) chaussettes

(D) Socken

\section{TIPPERARY}

Tipperary fortune (NC, 1785, ob.)

(cf. Cambridge fortune)

Tipperary lawyer (NC, 19-20)

(cf. Dublin dissector)

\section{TOLEDO}

Holy Toledo! (US, NC, 19-20)

(cf. Holy Jerusalem !)

\section{TRIPOLI}

to come from Tripoli (NC, 1847-90)

to fall

(F) tomber par terre

(D) auf die Erde fallen

\section{TROY}

Trojan

1. cf. Corinthian (17-18) 2. decent fellow

(1660) 3. brave man (1838)

(F) 2. chic type 3. homme brave

(D) 2. fabelhafter Kerl 3. mutiger Mensch

Trojan work (19)

hard work

(F) travail pénible; travail de Romain (1907)

(D) beschwerliche Arbeit

like a Trojan (1846)

relentlessly

(F) vaillamment

(D) tüchtig

trusty Trojan (17)

reliable, trustworthy person

(F) homme loyal, à qui I'on peut faire confiance

(D) zuverlässiger Mensch

\section{TURKEY}

Turk

1. cruel man (1536) 2. strong man 3.

Irishman (US, 20) 4. cf. Prussian

(F) 1. homme cruel; $\operatorname{Turc}(17,0 b) 2$.

homme fort; Turc 3. Irlandais 4. cf. Prussian

(D) 1. grausamer Mensch 2. starker M ann 3. Ire 4. cf. Prussian

Turks (NC, 1949)

the Irish

(F) les Irlandais

(D) die Iren

Turk's head (NC, 1820, ob.)

ceiling brush

(F) tête-de-loup

(D) kugelförmiger Besen am langen Stiel

little Turk (19)

urchin, little terror

(F) enfant terrible, petit démon

(D) lästiges Kind

to turn Turk (19)

to fly into a temper

(F) faire le méchant

(D) böse tun

young Turks (19)

avant-garde of a political party

(F) avant-garde d'un parti politique; jeunes

Turcs (1908)

(D) Avantgarde einer politischen Partei ; Jungtürken

Turkish medal (NC, 1914, ob.)

(cf. Abyssinian medal)

Turkish treatment

ruthless, brutal treatment

(F) mauvais traitement

(D) schonungslose Behandlung

many-colored like a Turkish carpet (16)

gaudily clothed

(F) habillé de couleurs vives; bigarré comme

un tapis de Turquie (1640)

(D) buntgekleidet

\section{VANDALS}

vandal (1663)

destroyer (of works of art)

(F) destructeur (de belles choses); vandale

(1733)

(D) Verwüster (von Kunstsachen); Vandale

Vandalism (1787)

wilful destruction

(F) esprit de destruction, action de détruire; vandalisme (1739)

(D) Verwüstung(sgeist) ; Vandalismus 


\section{VATICAN}

Vatican roulette (US, NC, 20)

rhythm birth control

(F) méthode naturelle de contraception

(D) natürliche Geburtenbeschränkung

\section{VERMONT}

Vermont green (US, NC, 20)

(cf. Acapulco)

\section{VIRGINIA}

Virginia weed (US, NC, 1821-65)

tobacco

(F) tabac

(D) Tabak

kerbside Virginia (NC, 20)

cigarette made from fag-ends

(F) cigarette faite de mégots

(D) Zigarette aus Stummel gemacht

\section{WALES}

Welsh comb (NC, 1785-1840)

the thumb and four fingers

(F) le pouce et les quatre doigts; peigne de

I'Allemand (17, ob.)

(D) der Daumen und die vier Finger

Welsh cricket (NC, 16-17)

(cf. Arkansas lizard)

Welsh fiddle (NC, 17-19)

(cf. Scoth fiddle)

Welsh goat (NC, 18-19)

Welshman

(F) Gallois

(D) Einwhoner von Wales

Welsh parlsey (NC, 1620-50)

hemp

(F) chanvre

(D) Hanf

like a Welsh mile, long and narrow (NC, 17851850)

said of anything so shaped

(F) se dit de toute chose longue et étroite

(D) bezeichnet irgendwelche lange, schmale Sache

to make a Welshman's hose of sthg, to turn sthg like a Welshman' hose (NC, 1520-1600)

to turn things to one's advantage

(F) tourner les choses à son avantage

(D) die Sachen seinen eigenen Zwecken zuwenden

\section{WARWICK}

Warwick Farms (RS, 20)

arms

(F) bras

(D) Arme

\section{WASHINGTON}

bird of Washington (US)

the American eagle
(F) l'aigle (de l'emblème) américain

(D) der Adler vom amerikanischen Wappen-

bild

\section{WATERBURY}

Waterbury watch (RS, 1932)

Scotch whisky

(F) whisky écossais

(D) schottischer Whisky

\section{WATERLOO}

to meet one's Waterloo

to suffer a decisive defeat

(F) subir une défaite décisive; rencontrer son Waterloo

(D) eine entscheidende Niederlage erleiden

\section{WEST HAM}

West $\mathrm{H}$ am reserves $(\mathrm{RS}, 20)$

nerves

(F) nerfs

(D) Nerven

\section{WESTPHALIA}

Westphalia (NC, 1904, ob.)

(cf. Brunswick 1)

\section{WINDSOR}

Old Brown Windsor (NC, 1942)

(cf. Brunswick 2)

\section{WINCHESTER}

Winchester goose/pigeon (NC, 1570)

(cf. French pig)

\section{WORMS}

to be/have gone to the diet of Worms (NC,

1760-1830)

to be dead and buried

(F) être mort et enterré

(D) tod und begraben sein

\section{YARMOUTH}

Yarmouth bee (NC, 19-20, ob.)/capon (NC, 1660, ob.)

(cf. Glasgow magistrate)

Yarmouth bloater

1. native or inhabitant of Yarmouth (NC, 1850) 2. motor-car (RS, 1910)

(F) 1. natif ou habitant de Yarmouth 2. automobile

(D) 1. Eingeborener bezw. Einwohner von Yarmouth 2. Auto

gone Yarmouth/quite Yarmouth (NC, 20)

YORK

(cf. Lakes of Killarney)

Duke of York (RS)

1. to walk (1859) 2. to talk (1873) 3. cork

(1890) 4. chalk (20)

(F) 1. marcher 2. parler 3. bouchon 4. craie

(D) 1. gehen, spazieren 2. sprechen 3. Korken

4. Kreide 
Duke of Yorks (RS)

1. forks (1874) 2. fingers, hands (1874)

(F) 1. fourchettes 2. doigts, mains

(D) 1. Gabeln 2. Finger, Hände

\section{YORKSHIRE}

Yorkshire (19-20)

cajolery

(F) duperie par des paroles captieuses,

enjôlement

(D) Beschwatzen

Yorkshire bite

1. cute piece of overreaching (1795) 2. sharp person (1801)

(F) 1 . fourberie astucieuse 2. individu retors, peu scrupuleux

(D) 1. übler Streich 2. geriebener, skrupelloser Kerl

Yorkshire compliment (19-20, ob.)

gift useless to giver and not wanted by receiver

(F) cadeau d'un objet inutile au donneur et non désiré par le bénéficiaire

(D) Geschenk einer dem Geber nutzlosen und vom Empfänger unerwünschten Sache

Yorkshire estate (19-20, ob.)

money in prospect

(F) perspectives d'argent

(D) Geldaussichten
Yorkshire reckoning (19-20)

(cf. Chinaman's shout)

Yorkshire tyke

1. native or inhabitant of Yorkshire (NC, 17-

20) 2. microphone (RS, 1945)

(F) 1. natif ou habitant du Yorkshire 2.

microphone

(D) Eingeborener bezw. Einwohner vom Yorkshire 2. Mikrophon

confident as a Yorkshire carrier (18-20, ob.) self-assured

(F) sûr de soi

(D) selbstbewusst

to come Yorkshire on (19-20)/over (1785) s.0., to put Yorkshire (up) on s.o. (18-20)

(cf. to jew)

to go Yorkshire (19-20)

(cf. to go Dutch)

when I come into my Yorkshire estates (19-20,

ob.)

when I have the money

(F) quand j'aurai les moyens

(D) wann ich mal die Mittel habe

he's as cunning as a Yorkshireman

he's a sly fox

(F) il est rusé; c'est un fin Normand

(D) er ist ein schlauer Fuchs; alter Schwede

(NC, 18) 


\section{LEXIQUE D'EXPRESSIONS ALLEMANDES}

\section{ABESSINIEN}

Abessinien (NC, 1925)

Nacktbadestrand

(F) plage naturiste

(E) nudist beach

Abessinier (NC, 1953)

Nacktbadender

(F) nudiste, naturiste

(E) nudist

\section{AEGYPTEN}

ägyptische Finsternis

absolute Finsternis

(F) obscurité totale; combat de nègres dans

un tunnel

(E) utter darkness

sich nach den Fleischtöpfen Aegyptens sehnen

sich nach einem früheren Wohlleben sehnen

(F) regretter une situation antérieure;

regretter les oignons d'Égypte

(E) to regret a former situation; to long/sigh for the fleshpots of Egypt

\section{AMERIKA}

damit wirst du nicht Amerika machen

das wird nicht viel abwerfen

(F) cela ne rapportera pas beaucoup; ce n'est

pas le Pérou (1867)

(E) it's not very profitable

einen reichen Onkel in Amerika haben

Aussicht auf eine Erbschaft haben

(F) avoir des espérances d'héritage; avoir un oncle d'Amérique (1826)

(E) to have a rich uncle

amerikanische Einladung

eine wozu jeder seinen Anteil beiträgt

$(F)$ invitation à laquelle chacun apporte son écot; invitation à l'américaine

(E) an invitation where each pays his part;

Chinaman's shout (NC, 20), Dutch party

(20)/treat (1875), Yorkshire reckoning (19-20)

ARANJUEZ

die schönen Tage von Aranjuez sind zu Ende

(cf. sich nach den Fleischtöpfen Aegyptens sehnen)

\section{ARKADIEN}

arkadisch

einfach ländlich, friedlich

(F) d'une simplicité pastorale, idyllique; arcadien

(E) ideally rustic; idyllic; Arcadian (1667)

\section{ATTIKA}

attisches Salz

beissender Witz
(F) esprit caustique; sel attique

(E) caustic wit; Attic salt/wit (17)

\section{ATHEN}

Eulen nach Athen tragen

Unnötiges, Ueberflüssiges tun

(F) faire qqch d'inutile ou de ridiculement superflu; vendre des coquilles à ceux qui viennent de Saint-M ichel (16)

(E) to do sthg useless or stupid; to carry coals to Newcastle (16-20)

\section{BABYLONIEN}

babylonisch

prunkvoll

(F) pompeux, fastueux

(E) pompous

\section{BARCELONA}

jede Menge Barcelona (NC, 1935)

jede beliebige $\mathrm{M}$ enge

(F) une quantité quelconque

(E) any quantity

BAYERN

bayerische Banane (NC, 1925)

Weisswurst

(F) boudin blanc

(E) white pudding

\section{BERBER}

Berber (NC, 1950)

alter Mann

(F) vieillard

(E) old man

zeitarmer Berber (NC, 1959)

der eine Verabredung nicht einhält

(F) qqn qui ne respecte pas un rendez-vous

(E) one who does not keep an appointment

\section{BERLIN}

Berliner (NC, 1950)

Jungmädchenbusen

(F) poitrine plate (de jeune fille)

(E) (young girl's) flat breasts

\section{BETTENHAUSEN}

nach Bettenhausen gehen (NC, 1880)

zu Bett gehen

(F) aller au lit

(E) to go to bed; to go to Bedfordshire (NC,

17-20, ob.)

\section{BOHEMEN}

Boheme (wesen) (19)

sorglose, unsichere Künstlerwelt

(F) vie d'artiste non conventionnelle; bohème (1838)

(E) unconventional artists's life; Bohemia (1861) 
Bohemien (19)

verbummeltes Genie

(F) (artiste) qui vit au jour le jour ; bohème (1838)

(E) one who leads an irregular life; bohemian (1848)

das kommt mir böhmisch vor (NC, 17)

das ist verdächtig, zweifelhaft

(F) cela me semble suspect

$(E)$ that's rather shady

das sind mir böhmische Dörfer (NC, 16-17)

es ist für mich Unverständliches

(F) cela m'est inintelligible; c'est de

I'allemand, du haut-allemand (17)/de

l'auvergnat (NC, 20)/du chinois (1790)/du

grec (17)/de l'hébreu (1530)/de l'iroquois (19) pour moi

(E) I don't understand a word; that's all

Dutch (NC, 18-20)/Greek (NC, 17-20) to me

ein Bohemeleben führen (19)

ein unkonventionelles Leben führen

(F) mener une vie insouciante et précaire;

mener une vie de bohème (1838), vivre

comme un bohème

(E) to lead a careless, unconventional life; to

\section{BONN}

lead a Bohemian life (1861)

Bonner Volkswagen (NC, 1950)

grosser M ercedes 300

(F) grosse voiture Mercedes 300

(E) big M ercedes-300 car

\section{BRABANT}

das kostet Holland und Brabant

das kostet eine Menge Geld

(F) cela coûte beaucoup d'argent

(E) that costs a lot of money

\section{BUXTEHUDE}

in Buxtehude wohnen (NC, 1850)

in einem kleinen Dorf, aus der Welt wohnen

(F) habiter un endroit reculé; habiter à

Fouilly-les-Oies

(E) to live in a remote place; to live in Hicksville (US, NC)

jemanden nach Buxtehude wünschen (NC, 19)

jemanden zum Teufel wünschen

(F) envoyer qqn au diable; envoyer qqn à Cancale (17)/à Pampelune (17)/se faire voir chez les Grecs (NC, 20)

(E) to wish S.o. to hell; to wish S.o. to Jericho (NC, 17)

\section{CASABLANCA}

Casablanca (NC, 1914-18)

kein Geld haben

(F) complètement démuni

(E) broke

\section{CHINA}

so was lebt in China!

Ausruf der Verwunderung

(F) exclamation de surprise

(E) exclamation of surprise; Jerusalem ! (NC,

19-20), Holy Jerusalem! (US, NC, 20), Holy

Toledo! (US, NC, 19-20)

lieber einen toten Chinesen im Arm als so aussehen wie du! (NC, 20)

Herabwürdigung des Aussehens eines anderen

(F) dénigrement de l'apparence d'autrui

(E) disparagement of someone's looks

Pappchinese (NC, 1910)

Schimpfwort

(F) injure

(E) term of abuse

chinesischer Spinat (NC, 1920)

Kuhfladen

(F) bouse de vache

(E) cow dung

das ist chinesisch für mich!

(cf. das sind mir böhmische Dörfer)

\section{DAMASKUS}

seinen Tag von Damaskus erleben

sich plötzlich bekehren, anderen Sinnes werden

(F) se convertir, changer d'opinion; trouver

son chemin de Damas

(E) to convert oneself, to change one's mind

\section{DAENEMARK}

etwas ist faul im Staate Dänemark (1850)

es stimmt hier was nicht

(F) il y a qqch de louche dans cette affaire; il

y a qqch de pourri dans le royaume de

Danemark (17)

(E) there is sthg shady in this business;

something is rotten in the state of Denmark

(17)

\section{DARMSTADT}

Gruss/Telegramm aus Darmstadt (NC, 19) entweichender Wind

(F) pet

(E) flatus

\section{DAVOS}

letzte Grüsse aus Davos (NC)

kränkliches Husten

(F) toux maladive

(E) ill-boding cough; Arizona tenor (US,

NC)

\section{DEUTSCHLAND}

deutsche Ananas (NC, 20)

Kohlrübe

(F) chou-navet

(E) swede (1812) 
deutscher (Autofahrer) Gruss (NC, 1945)

Berühren der Stirn mit dem Zeigefinger

(F) geste de l'index sur la tempe (signe du dérangement mental)

(E) putting one's finger to one's temple (meaning crazy)

deutscher Blick (NC, 1933)

vorsichtig Umherblicken

(F) regard scrutateur; œil américain (NC,

1872)

(E) searching look

deutscher Kaviar (NC, 1914-18)

1. Heringsrogen 2. polierter Sago

(F) 1. œufs de hareng 2. tapioca

(E) 1. herring's roe corn 2. tapioca

deutscher Michel (NC)

Spiessbürger

(F) petit bourgeois allemand

(E) typical German middle-class man deutsch reden (15)

offen, unumwunden reden

(F) parler franchement; parler français (16)

(E) to speak frankly; to speak plain English

deutscher Urwaldtee (NC, 1914-18)

Kräuterteegemisch

(F) tisane de plantes

(E) infusion of herbs

altdeutsch beischlafen (NC, 1922)

normaler Koitus

(F) faire l'amour classiquement

(E) to have normal sex

altdeutsch reden

(cf. deutsch reden)

auf gut deutsch (16)

direkt und einfach gesagt

(F) sans détours; en bon français (16)

(E) straightaway; in plain English (17)

du verstehst wohl kein deutsch mehr ! du

verstehst wohl nicht mehr deutsch !

du willst wohl nicht hören

(F) tu n'écoutes donc pas!; tu ne comprends plus le français!

(E) won't you listen ?; don't you understand plain English?

Hochdeutsch mit Streifen (NC, 19)

unreines deutsch

(F) parler incorrect

(E) faulty speaking

M arke deutscher Wald (NC, 1914-18)

minderwertiger Tabak

(F) mauvais tabac

(E) low-grade tobacco

Papierdeutsch (NC, 1930)

umständliche Ausdrucksweise, geschraubter Stil
(F) parler affecté, style ampoulé

(E) affected speech, stilted style

Plattdeutsche (NC, 1920)

flachbusige Frau

(F) femme qui a peu de poitrine

(E) flat-breasted woman

so deutsch wie Münchner Bier

hundertprozentig deutsch

(F) Allemand jusqu'au bout des ongles

(E) staunch German

\section{ENGLAND}

Pelzmantel des Engländers (NC, 1900)

Hosentaschen des M annes, der beide Hände

in sie steckt

(F) poches du pantalon, dans lesquelles

I'homme garde les deux mains

(E) trouser pockets in which a man keeps

both his hands

(ein bischen) schief ist Englisch (NC, 19)

Superlativgeltung

(F) c'est du dernier chic

(E) that's real super

englisch einkaufen (NC, 1920)

stehlen

(F) voler

(E) to steal

\section{ESKIMO}

das haut den stärksten Eskimo vom Schlitten!

(NC, 1930)

Ausruf höchster U eberraschung

(F) exclamation de surprise

(E) exclamation of surprise; that beats the

Dutch! (NC, 19-20)

\section{FRANKREICH}

wie Gott in Frankreich leben (18)

herrlich, üppig leben

(F) vivre dans l'opulence

(E) to live in affluence

auf französisch Abschied nehmen,

sich französisch verabschieden

diskret, heimlich davongehen

(F) s'en aller discrètement; filer à l'anglaise (1857)

(E) to depart unobtrusively; to take Dutch

(US)/French leave (1770)

Franzosenkrankheit

Syphilis

(F) syphilis; mal espagnol (15)/français/ de Naples (17)

(E) syphilis; French ache/disease/fever/ goods/gout/measles/marbles/mole/ pox (NC, 17-19), Neapolitan disease (16-17), Spanish gout/needle/pox (NC, 17-19) 


\section{HANAKEN}

H anake (NC, 18)

Schurke, ehrlose Person

(F) coquin, fourbe

(E) rogue, swindler ; Tartar (NC, 16-18), Yorkshire bite (NC, 1801)

\section{HAVANNA}

Havanna mit Plünneneinlage (NC, 1936) minderwertige Zigarre

(F) mauvais cigare

(E) cheap cigar; M anila rope (US, NC)

\section{HOLLAND}

da ist Holland in Not! (18)

es besteht eine Not- bezw. Zwangslage

(F) il y a urgence

(E) it's a matter of urgency

das kostet $\mathrm{H}$ olland und Brabant!

(cf. Brabant)

\section{HORNBERG}

ausgehen wie das Hornberger Schiessen

ergebnislos enden

(F) n'aboutir à rien

(F) to come to nothing

\section{HOTTENTOTTEN}

H ottentottenboy (NC, 1955)

modisch gekleideter $\mathrm{Halbwüchsiger}$

(F) jeune gandin

(E) young dandy

es sieht aus wie bei den Hottentotten (NC, 20)

es ist ein richtiges Durcheinander

(F) grand désordre; c'est un vrai caphar-

naüm/une maison de bohème (19)

(E) it's a regular chaos

\section{HUNNEN}

wie die Hunnen hausen

wild toben, alles zerstören

(F) tempêter, saccager

(E) to rage and devastate

\section{INDIANER}

Indianergeschenk (NC)

Geschenk in Erwartung eines Gegen-

geschenks

(F) cadeau en échange d'un autre

(E) a gift in exchange for another; Indian gift (NC)

Plattfussindianer (NC, 1900)

1. Schimpfwort 2. Verkehrspolizist

(F) 1. insulte 2. agent de la circulation

(E) 1. term of abuse 2. traffic policeman

Schweissfussindianer (NC, 1914-18)

Infanterist

(F) fantassin

(E) infantryman

Vollblutindianer (NC, 1920)

sehr dummer $M$ ensch
(F) individu stupide; béotien

(E) simpleton; Bœotian (16), Hottentot (NC, 19)

\section{ITHAKA}

Ithaker (NC, 1940)

italienischer Soldat; Italiener

(F) soldat italien; Italien

(E) Italian (soldier)

JUDEN

Jude

Wucherer

(F) usurier; juif (17)

(E) usurer ; Jew (1606)

für's Gewesene gibt der Jude nichts

was weg ist beisst nicht mehr

(F) le moulin ne meut pas avec l'eau coulée

en bas

(E) water run by will not turn a mill

haust du meinen Juden, hau ich deinen Juden

mit gleicher Münze heimzahlen

(F) rendre la pareille

(E) to retaliate

Lärm wie in einer Judenschule (NC, 18)

1. grosser Lärm 2. Stimmengewirr

(F) 1. vacarme 2. brouhaha

(E) 1. big noise 2. hum (of loud voices)

unbeschnittener/weisser Jude (NC)

erfahrener christlicher Geschäftsmann

(F) homme d'affaires avisé (non juif)

(E) (non Jewish) shrewd businessman

nur keine jüdische $\mathrm{H}$ ast!

keine übertriebene Hast!

(F) pas de précipitation!

(E) easy does it!

\section{KAFFERNLAND}

Kaffer (NC, 18)

1. cf. Vollblutindianer

2. Flegel, Grobian

(F) rustre; ostrogoth (1690)

(E) boor; big Dutch(man) (US, NC), Goth

(1663), big Swede (US, NC)

\section{KANAKEN}

Kanake (NC, 1850)

(cf. Kaffer 2)

\section{KANOSSA}

den Kanossaweg antreten, nach Kanossa gehen demütig um Vergebung bitten

(F) s'incliner honteusement; aller à Canossa

(19)

(E) to eat humble pie; to go to Canossa (19)

\section{KASSEL}

ab nach Kassel ! (NC)

fort mit dir, verschwinde!

(F) disparais, va-t'en !; va te faire voir chez les Grecs! (NC, 20) 
(E) go away, be off !; go to Bath !(NC, 1830)/ to Hell or Connaught (NC, 1654)/to Halifax! (NC, 17-20)/to Jericho! (NC, 1635)/to Jerusalem! (NC, 19-20)

\section{KISSINGEN}

nach Kissingen gehen (NC, 1900)

(cf. nach Bettenhausen gehen)

\section{KOBLENZ}

noblenz Koblenz/nolens Koblenz (NC, 19) widerwillig

(F) bon gré, mal gré

(E) willy-nilly

\section{KORINTH}

Korinthenkacker (NC, 19)

kleinlicher Mensch

(F) individu mesquin

(E) mean person

\section{LEIPZIG}

Anno Leipzig/einundleipzig (NC, 1913)

vor langer Zeit

(F) il y a longtemps

(E) a long time ago

\section{LESBOS}

Lesbierin

Tribade

(F) tribade; lesbienne (1867)

(E) female homosexual ; lesbian (1900)

\section{LIMBURG}

Limburger (NC, 19)

Schweissfüsse

(F) pieds qui transpirent

(E) sweating feet

\section{LOMBARDEI}

Lombard

Pfandhaus

(F) mont-de-piété

(E) pawnshop; M oscow (NC, 20)

Lombarde

Geldwechsler/-verleiher

(F) changeur, prêteur ; lombard $(1709$, ob.)

(E) money-changer/-lender; lombard (17)

\section{MARATHON}

Marathon(lauf) (1896)

Wettlauf über 42,195 Km

(F) course à pied de 42,195 km; marathon

(E) marathon (race)

Marathonrede, Marathonsitzung (20)

von besonders langer Dauer

(F) discours/séance de longue durée;

marathon oratoire, séance marathon (20)

(E) long speech/session; marathon speech/ session

\section{METZ}

raus aus $M$ etz! (NC, 19)

\section{MOHIKANER}

der letzte M ohikaner (NC, 1900)

das Allerletzte (Stück Geld, Flasche, usw.)

(F) l'ultime exemplaire (billet de banque, bouteille, etc.) ; le dernier des M ohicans

(E) the very last piece (banknote, bottle, etc.)

\section{MOHREN}

der M ohre hat seine Schuldigkeit getan,

der Mohre kann gehen

sich ungerecht behandelt fühlen

(F) le monde paie d'ingratitude

(E) thanklessness is human

einen Mohren kann man nicht weiss waschen; das heisst einen $M$ ohren weiss waschen wollen (16)

etwas vergebliches versuchen

(F) tenter l'impossible; à laver un nère on perd son savon

(E) to attempt the impossible; you cannot wash a blackamoor white (16)

finster wie in einem M ohrenarsch stockdunkel

(F) dans l'obscurité totale; noir comme dans le trou du cul d'un nègre (NC, 19)

(E) in complete darkness

schwarz wie ein M ohrenkind absolut schwarz

(F) noir comme l'ébène

(E) as black as pitch

\section{MONGOLIEN}

Mongoloider

Kind mit Trisomie 21

(F) enfant atteint de trisomie 21; mongol(ien)

(E) child with trisomy 21 ; M ongolian child Mongolismus

Downsyndrom

(F) syndrome de Down ; mongolisme

(E) Down syndrome; Kalmuk/M ongolian idiocy, mongolism

\section{MÜNCHEN}

so deutsch wie Münchner Bier (cf. deutsch)

NASSAU

Nassauer (NC, 19)

1. Platzregen 2. Schmarotzer

(F) 1. averse 2. écornifleur

(E) 1. shower; Scotch mist (NC, 19-20)

2. parasite, cadger

OBERAMMERGAU

Oberammergauner (NC, 1925)

(cf. Hanake)

\section{OXFORD}

schick den Esel nach Oxford, nimmer wird ein Pferd er dort durch reisen verbessert man sich nicht 
(F) fou va à Rome, fou en revient; jamais cheval ni méchant homme n'amenda pour aller à Rome; qui bête va à Rome, tel en retourne

(E) send a fool to the market and a fool he'll return

\section{PAPPENHEIM}

seine Pappenheimer kennen (NC, 1892) die Leute (Freunde, Nachbarn, usw.) durchaus kennen

(F) savoir à qui I'on a affaire

(E) to know whom one is dealing with

\section{PARIS}

Pariser (NC, 19)

Präservativ

(F) préservatif; capote anglaise (NC, 1870)

(E) condom; American (NC, 19)/French

(NC, 1870)/Italian (NC, 18-20)/ Spanish

(NC, 18-20) letter, Manhattan eel (US, NC,

20), Port Said garter $(N C, 20)$

\section{PHILISTER}

Philister (19)

1. Zivilist 2. Mensch mit sehr konservativen

Ansichten

(F) 1. civil, pékin (1799) 2. bourgeois peu

éclairé; philistin (1832)

(E) 1. civilian 2. a person deficient in liberal

\section{POLEN}

culture; philistine (1827)

noch ist Polen nicht verloren

noch besteht Hoffnung

(F) tout espoir n'est pas perdu

(E) the day is not yet lost

polnische Wirtschaft (NC, 1835)

(cf. es sieht aus wie bei den Hottentotten)

\section{PREUSSEN}

so schnell schiessen die Preussen nicht!

ein bischen Geduld!

(F) un peu de patience!

(E) (have) patience!

preussische Beilage (NC, 1962)

Kartoffeln

(F) pommes de terre; canadas

(E) potatoes; Irish apples (NC, 19)/ apricots

(NC, 1815)/grapes (US, NC, 19)/lemons

(NC, 19), M unster plums (NC, 1780)

\section{ROM}

Rom ist nicht an einem Tage erbaut worden das Ziel lässt sich nicht sofort erreichen

(F) les choses doivent se faire avec patience; Paris/Rome ne s'est pas fait(e) en un jour (16-17)

(E) patience is needed to achieve things; Rome was not built in a day (16) alle Wege führen nach Rom das Ziel lässt sich auf verschiedene Weisen erreichen

(F) il y a de nombreux moyens d'arriver au but; tous les chemins mènent à Rome

(E) there are many ways to achieve an end ; all roads lead to Rome

auf diesem Messer kann man bis/nach Rom reiten

dieses M esser ist sehr stumpf

(F) ce couteau ne coupe pas

(E) this knife has no edge; you can ride to Romford on this knife

er war in Rom und hat den Papst nicht gesehen er hat das wichtigste nicht bemerkt

$(F)$ il a raté l'essentiel

(E) he missed the main thing

in Rom, tu' wie Rom tut

man soll sich nach den Bräuchen des Landes richten

(F) il faut se conformer aux usages du pays; à Rome, il faut vivre comme à Rome

(E) one should comply with local usage; at Rome you must do as the Romans do, when at Rome do as the Romans do

lieber der erste hier als der zweite in Rom eine örtliche Berümtheit ist besser

(F) mieux vaut être connu chez soi ; il vaut mieux être le premier dans son village que le second dans Rome

(E) a local fame is better; better to reign in $\mathrm{Hell}$ than to serve in Heaven

Zustände wie im alten Rom ein unhaltbarer, unmöglicher Zustand

(F) situation impossible, intenable

(E) impossible, intolerable situation

auf so etwas hätten die alten Römer geschossen das ist eine höchst minderwertige Sache

(F) cela ne vaut absolument rien

(E) that's absolutely worthless

\section{RUSSLAND}

jemanden einen Russen aufbinden (NC, 1945) jemandem Unwahres zu glauben geben

(F) en faire accroire à qqn

(E) to spin a yarn to s.o.

scharf wie tausend Russen (NC, 1942)

sehr geil

$(F)$ très porté sur le sexe

(E) very fond of sex

\section{SAMARIEN}

den barmherzigen Samariter spielen mitleidig sein

(F) être secourable; jouer au bon Samaritain

(E) to be compassionate; to be a good

Samaritan 


\section{SCHAFFH AUSEN}

Rheinfall bei/von Schaffhausen (NC, 1875)

Betrug

(F) tromperie

(E) cheating, swindle

SCHWABEN

ins Schwabenalter kommen (NC, 1773)

40 Jahre alt werden

(F) franchir le cap de la quarantaine

(E) to reach the forty-mark

\section{SCHWEDEN}

alter Schwede (NC, 18)

gerissener $M$ ensch

(F) individu rusé; fin Normand

(E) sly character; as cunning as a Yorkshireman (19-20)

hinter schwedischen Gardinen sitzen (NC, 19)

im Gefängnis sitzen

(F) être en prison

(E) to be in prison; to be in Lombard Street (NC, 1810-60)

\section{SIBIRIEN}

lieber ein Jahr in Sibirien ohne Hose! (NC, 1935)

Abweisung einer Zumutung

(F) exclamation de refus

(E) an exclamation of refusal

\section{SODOM}

es ist ein wahres Sodom und Gomorra

ein Ort der grössten Unmoral

(F) c'est un lieu de corruption; c'est Sodome

et Gomorrhe

(E) it's an immoral, corrupt place; it's a regular Sodom

Sodomiterei

\section{Analkoitus}

(F) coït anal ; botte florentine (1789), sodomie

(E) anal coition; Bucklebury (NC, 1923), the Greek fashion/way (US, NC, 1940), Greek love (US, NC, 20)

\section{SPANIEN}

Spanien (NC, 1953)

Schulabort

(F) toilettes d'école

(E) school toilets

das kommt mir spanisch vor (17)

(cf. das kommt mir böhmisch vor)

jemandem spanisch kommen (NC, 1900)

mit dem Stock prügeln

(F) donner la bastonnade à qqn

(E) to cudgel s.o.

jemandem spanische Dörfer sein (18)

(cf. das sind mir böhmische Dörfer)

stolz wie ein Spanier

sehr stolz
(F) très fier; fier comme un Écossais

(E) very proud

\section{SPEYER}

nach Speyer appellieren (NC, 19)

sich erbrechen

(F) vomir

(E) to vomit; to go to Europe with Ralph

and Earl in a Buick (US, NC, 20)

\section{TROJA}

das Ross von Troja war gegen inn ein Zwergfüllen (NC)

er ist sehr dumm

(F) il est très bête

(E) he is very stupid

ein Geschenk wie das trojanische Pferd

ein betrügerisches Geschenk

(F) cadeau empoisonné

(E) treacherous gift; Grecian/Greek gift

\section{TUERKEI}

Türke (NC)

1. bis zum U eberdruss Wiederholtes

2. Hotelportier

(F) 1. répétition lassante 2 . portier d'hôtel

(E) 1 . sthg repeated ad nauseam 2 . hall porter

einen Türken bauen (NC, 1914-18)

1. etwas vortäuschen 2. Ehrenbezeugungen vollführen

(F) 1. simuler qqch 2. rendre les honneurs

(E) 1. to feign sthg 2. to pay honours

Kümmeltürke (NC)

1. wackerer Zecher 2. langweiliger Mensch

(F) 1 . ami de la dive bouteille 2. personne ennuyeuse

(E) 1. heavy drinker ; philistine 2. boring person

Kruzitürken ! (NC, 19)

ein Fluch

(F) juron

(E) swear-word; Holy Jerusalem! (US, NC, 20), Holy Toledo! (US, NC, 19-20)

\section{VANDALEN}

Vandale

Verwüster (von Kunstsachen)

(F) destructeur (de belles choses) ; vandale

(1732)

(E) destroyer (of works of art) ; vandal

(1752)

Vandalismus

Verwüstung(sgeist)

(F) esprit de destruction, action de détruire; vandalisme (1739)

(E) wilful destruction; vandalism (1787) 


\section{WELSCHLAND}

Kauderwelsch reden

Unverständliches reden

(F) parler un langage incompréhensible;

parler petit nègre (1857)

(E) to talk gibberish; to talk double-Dutch

(NC, 1860)

\section{WIEN}

Geschichten aus dem Wienerwald erzählen übertriebene, unglaubliche Geschichten erzählen

(F) raconter des histoires incroyables; être de M arseille; raconter des histoires de Gascon/ des histoires marseillaises

(E) to tell tall stories; to tell Texan tales (US, $\mathrm{NC)}$ 